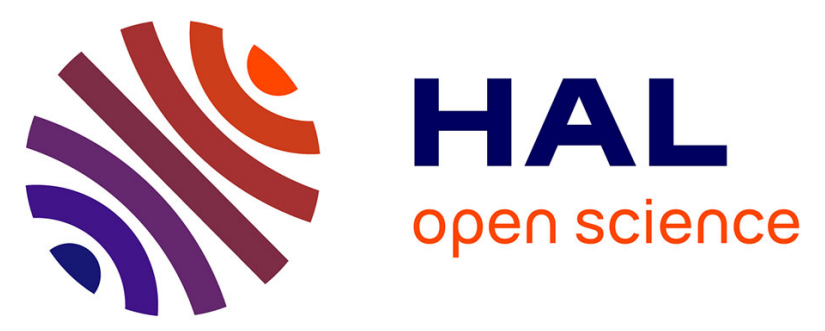

\title{
Martian oxygen density at the exobase deduced from OI 130.4-nm observations by Spectroscopy for the Investigation of the Characteristics of the Atmosphere of Mars on Mars Express
}

Jean-Yves Chaufray, François Leblanc, Eric Quémerais, Jean-Loup Bertaux

\section{To cite this version:}

Jean-Yves Chaufray, François Leblanc, Eric Quémerais, Jean-Loup Bertaux. Martian oxygen density at the exobase deduced from OI 130.4-nm observations by Spectroscopy for the Investigation of the Characteristics of the Atmosphere of Mars on Mars Express. Journal of Geophysical Research. Planets, 2009, 114 (E2), pp.E02006. 10.1029/2008JE003130 . hal-00363134

\section{HAL Id: hal-00363134 \\ https://hal.science/hal-00363134}

Submitted on 1 Feb 2016

HAL is a multi-disciplinary open access archive for the deposit and dissemination of scientific research documents, whether they are published or not. The documents may come from teaching and research institutions in France or abroad, or from public or private research centers.
L'archive ouverte pluridisciplinaire HAL, est destinée au dépôt et à la diffusion de documents scientifiques de niveau recherche, publiés ou non, émanant des établissements d'enseignement et de recherche français ou étrangers, des laboratoires publics ou privés. 


\title{
Martian oxygen density at the exobase deduced from $O$ I 130.4-nm observations by Spectroscopy for the Investigation of the Characteristics of the Atmosphere of Mars on Mars Express
}

\author{
J. Y. Chaufray, ${ }^{1,2}$ F. Leblanc, ${ }^{1,3}$ E. Quémerais, ${ }^{1}$ and J. L. Bertaux ${ }^{1}$ \\ Received 26 February 2008; revised 3 June 2008; accepted 24 September 2008; published 19 February 2009.
}

[1] Several observations of the O I 130.4-nm triplet have been analyzed to determine the oxygen density in the Martian upper atmosphere using a three-dimensional Monte Carlo radiative transfer model describing each line of the triplet. Solar resonant scattering is the dominant source of excitation of the O I 130.4-nm triplet in the upper atmosphere of Mars. The atomic oxygen density at the exobase is found to be $1.2_{-0.5}^{+1.2} \times 10^{7} \mathrm{~cm}^{-3}$ for solar zenith angles between $20^{\circ}$ and $55^{\circ}$ and to decrease by a factor of 2 for solar zenith angles between $55^{\circ}$ and $90^{\circ}$. Although the major contribution to the observed brightness is produced above the exobase, it is possible to extrapolate the density profile below the exobase and to estimate the $[\mathrm{O}] /\left[\mathrm{CO}_{2}\right]$ mixing ratio as $0.6-1.2 \%$ at $135 \mathrm{~km}$. The major source of uncertainty comes from the uncertainty in the absolute calibration, as expected for an optically thick emission, and also, to a lesser degree, from the temperature at the exobase. The profiles are better reproduced by a large exospheric temperature $(>300 \mathrm{~K})$, which may suggest the presence of a hot oxygen population.

Citation: Chaufray, J. Y., F. Leblanc, E. Quémerais, and J. L. Bertaux (2009), Martian oxygen density at the exobase deduced from O I 130.4-nm observations by Spectroscopy for the Investigation of the Characteristics of the Atmosphere of Mars on Mars Express, J. Geophys. Res., 114, E02006, doi:10.1029/2008JE003130.

\section{Introduction}

[2] The 130.4-nm oxygen triplet is a common UV airglow emission of the terrestrial planets. It was observed for the first time in Earth's upper atmosphere through rocket measurements [Chubb et al., 1958] but was also seen in the Venusian upper atmosphere [Moos and Rottman, 1971; Broadfoot et al., 1974; Bertaux et al., 1981] and in the Martian upper atmosphere [Barth et al., 1971; Feldman et al., 2000]. The first analyses of the Martian O I 130.4-nm triplet observed by the Mariner missions during high solar activity concluded that two mechanisms contribute to this emission: resonant scattering of solar photons and photoelectron impact excitation of atmospheric oxygen atoms [Strickland et al., 1972, 1973; Fox and Dalgarno, 1979]. The resonant scattering of the solar photons was shown to be the major source of excitation. Twenty years later a reanalysis of this emission was done [Stewart et al., 1992] using a more accurate radiative transfer model [Meier and Lee, 1982]. The photoelectron impact frequency was revised using the updated cross sections from Zipf and Erdman [1985] as well as an analytical relation between the $F_{10.7}$ solar activity index and the solar flux at $130.4 \mathrm{~nm}$. Two thermospheric models were used in the study by Stewart et al. [1992]: an empirical thermospheric model

\footnotetext{
${ }^{1}$ Laboratoire Atmospheres, Milieux, Observations Spatiales, IPSL, CNRS, Verrières-le-Buisson, France.

${ }^{2}$ Now at Southwest Research Institute, San Antonio, Texas, USA.

${ }^{3}$ Temporarily at Osservatorio Astronomico di Trieste, Trieste, Italy.

Copyright 2009 by the American Geophysical Union. 0148-0227/09/2008JE003130
}

[Stewart, 1987] and a Mars Thermosphere General Circulation Model (MTGCM) [Bougher et al., 1990]. The first model was used to reproduce the intensity by fitting the oxygen density, and the second model was used in order to reproduce the latitude and local time distribution. A mixing ratio $[\mathrm{O}] /\left[\mathrm{CO}_{2}\right]$ of $0.7 \%$ at the 1.2 -nbar level was found to provide the best fit of the measured intensity profile, while a ratio equal to $0.2 \%$ was found to provide the best fit of the latitude and local time variations. These ratios were slightly smaller than the earlier estimate of $1 \%$ by Strickland et al. [1972, 1973]. Another estimate of this ratio was made using Viking descent probe data obtained at low solar activity. Using a simple photochemical model, Hanson et al. [1977] calculated the best oxygen density profile able to reproduce the ion profiles measured by Viking and deduced a ratio $[\mathrm{O}] /\left[\mathrm{CO}_{2}\right]$ equal to $1.25 \%$ at $130 \mathrm{~km}$. The $[\mathrm{O}] /\left[\mathrm{CO}_{2}\right]$ ratio is an important element in the global heat budget of the thermosphere. Indeed, atomic oxygen collisions with $\mathrm{CO}_{2}$ molecules are known to be effective in exciting the $\mathrm{CO}_{2}\left(\nu_{2}\right)$ vibrational state, resulting in an enhanced $\mathrm{CO}_{2} 15-\mu \mathrm{m}$ emission, which, in turn, leads to an enhanced cooling rate in the thermosphere [Bougher et al., 1994, 1999, 2000]. For example, a higher $[\mathrm{O}] /\left[\mathrm{CO}_{2}\right]$ mixing ratio was suggested by Keating et al. [1998] in order to explain the discrepancy between the temperature deduced from Mars Global Surveyor (MGS) aerobraking data and the temperature calculated by the MTGCM model of Bougher et al. [1997] (120 K versus $150 \mathrm{~K}$ at $130 \mathrm{~km}$ ).

[3] In this paper we present an analysis of the 130.4-nm triplet measured by the Spectroscopy for the Investigation of the Characteristics of the Atmosphere of Mars-ultraviolet spectrometer (SPICAM-UVS) instrument [Bertaux et al., 


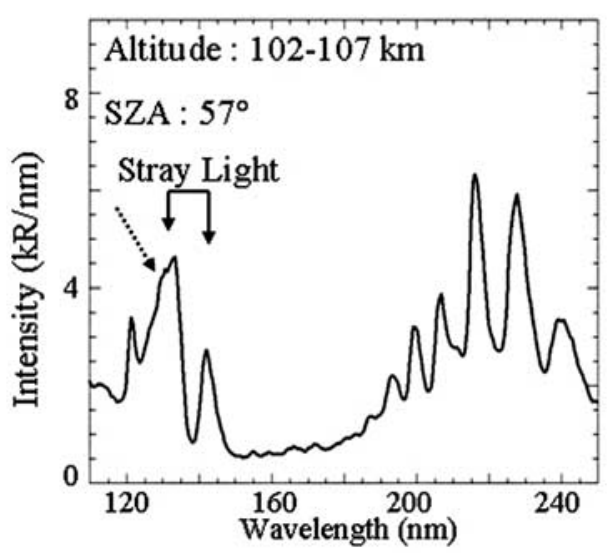

Figure 1. Example of average spectrum below $150 \mathrm{~km}$ obtained by Spectroscopy for the Investigation of the Characteristics of the Atmosphere of Mars-ultraviolet spectrometer (SPICAM-UVS). A stray light is observed centered near 132 and $143 \mathrm{~nm}$. The origin of this stray is still unknown. The dashed arrow indicates the expected position of the 130.4-nm lines. The oxygen $130.4 \mathrm{~nm}$ lines could correspond to the little bump on the left side of the stray light centered at $132 \mathrm{~nm}$. SZA, solar zenith angle.

2006] on board Mars Express in the Martian upper atmosphere. The observations presented in section 2 were obtained during moderate solar conditions [Leblanc et al., 2006]. The models of oxygen density, excitation sources, and radiative transfer are described in section 3 . The best oxygen density profiles and the uncertainties derived from a fit of the model to the observations are described in section 4 , and the results are discussed in section 5 .

\section{Observations}

[4] The SPICAM-UVS instrument is described in detail by Bertaux et al. [2006]. The observations used here are of
Martian limb [Bertaux et al., 2006] as seen near Mars Express pericenter from October 2004 to February 2005. The general data processing (dark current subtraction and integrated intensity calculation) is described by Leblanc et al. [2006]. Contrary to the approach of Leblanc et al. [2006, 2007] for the optically thin emissions, the oxygen $130.4 \mathrm{~nm}$ being optically thick, we find it unsuitable to average different observations on the basis of the altitude and solar zenith angle of the tangent point. To appropriately average O I 130.4-nm observations, the whole line of sight would have to be the same. However, the signal being rather weak (10 times weaker than the Lyman- $\alpha$ signal; see the work of Leblanc et al. [2006]), it remains necessary to add individual observations to increase the signal-to-noise ratio. Therefore the observations considered in this study were gathered into different groups (Table 1). Table 1 provides the main parameters of the observations for each group. Each group is divided into two subgroups corresponding to the upleg and downleg parts of the orbits. Here we consider only observations with a line of sight above $150 \mathrm{~km}$, because a strong solar scattering background has sometimes been identified at lower altitudes (particularly at low solar zenith angle). As an example, some of the observations presented here (group 2) were not considered by Leblanc et al. [2006], because a strong signal associated with stray light centered at 132 and $143 \mathrm{~nm}$ was present below $120 \mathrm{~km}$. This strong signal can overlap the hydrogen Lyman- $\alpha$ and the O 130.4-nm lines (Figure 1). Here we choose to keep these data because the stray light contribution does not seem to be present above $140 \mathrm{~km}$ (as determined by analyzing the Lyman- $\alpha$ profile).

[5] For each group, the profile of the O I 130.4-nm emission intensity from 150 up to $\sim 400 \mathrm{~km}$ has been calculated. Each point of each group profile is determined by averaging several spectra (between 11 and 65 individual spectra). Examples of average spectra are displayed in Figure 2 corresponding to groups $3 \mathrm{a}$ and $3 \mathrm{~b}$. As shown in Figure 2, the oxygen triplet intensity decreases as the altitude of the tangent point increases, while the Lyman- $\alpha$ line (at $121.6 \mathrm{~nm}$ )

Table 1. Groups of Orbits Analyzed in This Study ${ }^{\mathrm{a}}$

\begin{tabular}{|c|c|c|c|c|c|c|c|c|}
\hline & Date & $\begin{array}{c}\text { Altitude } \\
\text { (MEX, km) }\end{array}$ & $\begin{array}{c}\text { SZA } \\
(\mathrm{MEX})\end{array}$ & $\begin{array}{l}\text { Range of Altitude } \\
\text { (MNP, km) }\end{array}$ & $\begin{array}{c}\text { Range of SZA } \\
\text { (MNP, deg) }\end{array}$ & $F_{10.7}$ & $F_{130.4}$ & $L_{s}$ \\
\hline \multicolumn{9}{|c|}{ Group 1} \\
\hline First part $(947,948,952,958,959,969)$ & $15-21$ Oct 2004 & $520-810$ & $53-58$ & $150-390$ & $25-40$ & 32.3 & 3.9 & $101^{\circ}$ \\
\hline Second part $(947,948,952,958,959,969)$ & $15-21$ Oct 2004 & $280-390$ & $76-89$ & $150-360$ & $65-85$ & 32.3 & 3.9 & $101^{\circ}$ \\
\hline \multicolumn{9}{|c|}{ Group 2} \\
\hline First part $(1075,1077,1079,1080)$ & 19-21 Nov 2004 & $340-540$ & $24-39$ & $150-400$ & $54-56$ & 41.3 & 4.0 & $117^{\circ}$ \\
\hline Second part $(1075,1077,1079,1080)$ & 19-21 Nov 2004 & $350-540$ & $53-68$ & $150-400$ & $68-80$ & 41.3 & 4.0 & $117^{\circ}$ \\
\hline \multicolumn{9}{|c|}{ Group 3} \\
\hline First part $(1267,1271,1285)$ & 12-17 Jan 2005 & $360-540$ & $14-16$ & $150-400$ & $25-45$ & 36.6 & 4.2 & $144^{\circ}$ \\
\hline Second part $(1267,1271,1285)$ & $12-17$ Jan 2005 & $360-570$ & $59-63$ & $150-400$ & $55-72$ & 36.6 & 4.2 & $144^{\circ}$ \\
\hline \multicolumn{9}{|c|}{ Group 4} \\
\hline First part $(1349,1350,1357,1374)$ & 4-14 Feb 2005 & $720-1140$ & $4-9$ & $150-400$ & $22-32$ & 37.7 & 4.3 & $155^{\circ}-160^{\circ}$ \\
\hline Second part $(1349,1350,1357,1374)$ & $4-14$ Feb 2005 & $320-360$ & $54-61$ & $150-400$ & $54-90$ & 37.7 & 4.3 & $155^{\circ}-160^{\circ}$ \\
\hline \multicolumn{9}{|c|}{ Group 5} \\
\hline First part $(1413,1414)$ & $22 \mathrm{Feb} 2005$ & $360-560$ & $30-49$ & $150-400$ & $20-45$ & 54.2 & 4.8 & $164^{\circ}$ \\
\hline Second part $(1413,1414)$ & 22 Feb 2005 & $370-560$ & $79-97$ & $150-400$ & $77-100$ & 54.2 & 4.8 & $164^{\circ}$ \\
\hline
\end{tabular}



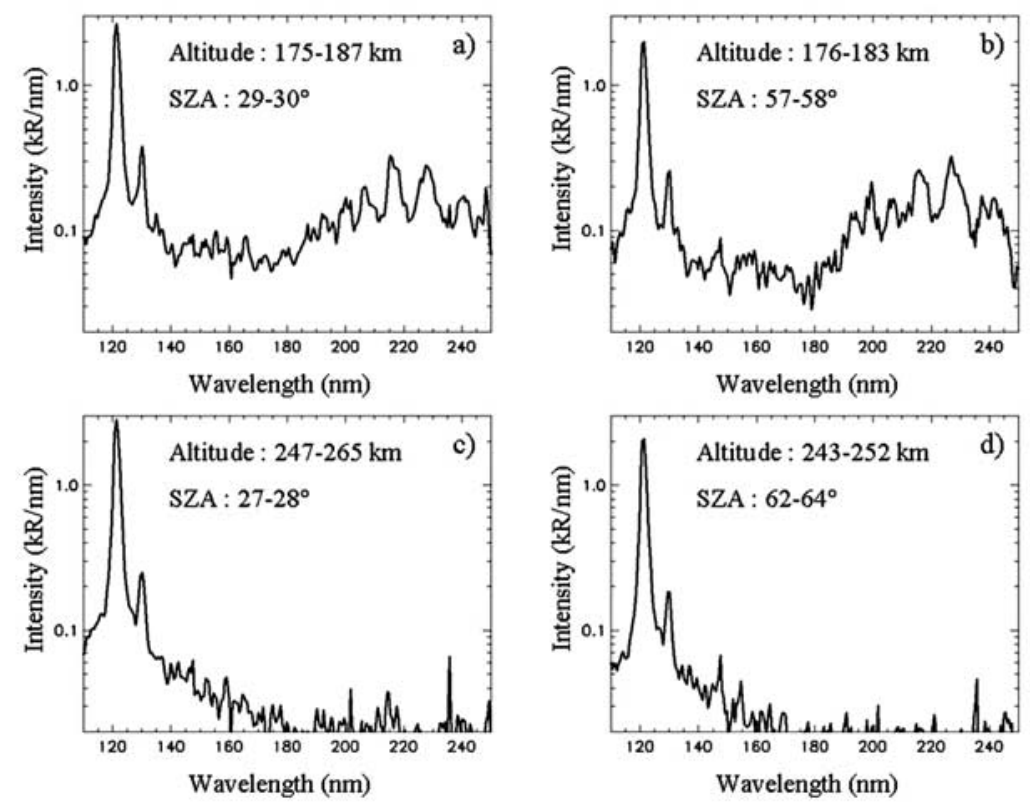

Figure 2. Examples of average spectra near (a, b) $150 \mathrm{~km}$ and (c, d) $240 \mathrm{~km}$. Spectra were obtained by SPICAM-UVS at solar zenith angles between $27^{\circ}$ and $30^{\circ}$ for Figures $2 \mathrm{a}$ and $2 \mathrm{c}$ and at solar zenith angles between $57^{\circ}$ and $64^{\circ}$ for Figures $2 \mathrm{~b}$ and $2 \mathrm{~d}$. Each average spectrum corresponds to $\sim 40-60$ individual integrations of SPICAM-UVS. The oxygen triplet is the line at $130 \mathrm{~nm}$. The Lyman- $\alpha$ line is also visible at $121.6 \mathrm{~nm}$. These spectra correspond to groups $3 \mathrm{a}$ and $3 \mathrm{~b}$.

does not change. On the contrary, less intense O I 130.4-nm and $\mathrm{H}$ I Lyman- $\alpha$ emissions are observed with increasing solar zenith angle (SZA) (when comparing Figures $2 \mathrm{a}$ and $2 \mathrm{~b}$ or Figures $2 \mathrm{c}$ and $2 \mathrm{~d}$ ). Figure 3 displays an example of intensity profile showing how the $\mathrm{O}$ emission scale height changes with respect to the solar zenith angle.

\section{Models of the Thermospheric and Exospheric Oxygen Densities and of the 130.4-nm Emission}

[6] In order to retrieve the atomic oxygen profile which best fits the observations, we have developed a model to calculate the O I 130.4-nm emission brightness. A model describing the oxygen and carbon dioxide density profiles has been developed (section 3.1) and has been coupled to a radiative transfer model (section 3.2).

\subsection{Oxygen Density Profiles From 80 to 1000 km}

[7] The density profiles are divided into two parts: a thermospheric profile from 80 to $200 \mathrm{~km}$ and an exospheric profile from 200 to $1000 \mathrm{~km}$. The $\mathrm{O}$ and $\mathrm{CO}_{2}$ thermospheric density profiles are calculated by solving the coupled diffusion and hydrostatic equations [Hunten, 1973]. In this region, the temperature and eddy mixing coefficient profiles $K(z)$ are taken from Krasnopolsky [2002]. The exospheric temperature is assumed to be equal to $200 \mathrm{~K}$ as derived from an analysis of the dayglow emissions associated with the $\mathrm{CO}_{2}^{+}$ and Vegard-Kaplan $\mathrm{N}_{2}$ bands by Leblanc et al. [2007], in good agreement with the solar minimum thermosphere model of Krasnopolsky [2002]. The $\mathrm{CO}_{2}$ number density at $80 \mathrm{~km}$ is chosen to be $2.6 \times 10^{13} \mathrm{~cm}^{-3}$ [Krasnopolsky, 2002].
The diffusion coefficient $D_{O}$ of $\mathrm{O}$ atoms through a $\mathrm{CO}_{2}$ atmosphere depends on the temperature:

$$
D_{O}(r)=\frac{A T(r)^{s}}{n(r)}
$$

where $n(r)$ is the total local density $\left(\mathrm{O}+\mathrm{CO}_{2}\right), T(r)$ is the local temperature [from Krasnopolsky, 2002], $A$ is equal to

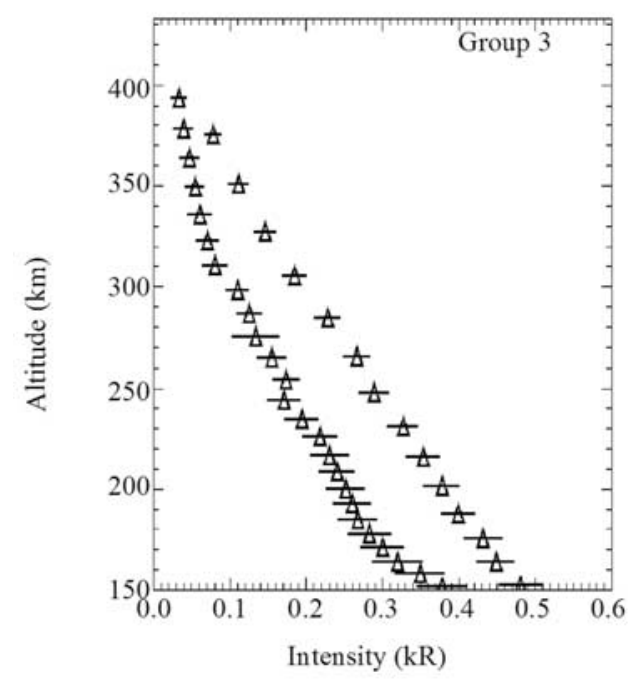

Figure 3. Altitude profiles of the $\mathrm{O} 130.4-\mathrm{nm}$ emission intensity at high SZA (left curve) and low SZA (right curve) for group 3 (see Table 1 for the definition and observation parameters of this group). 


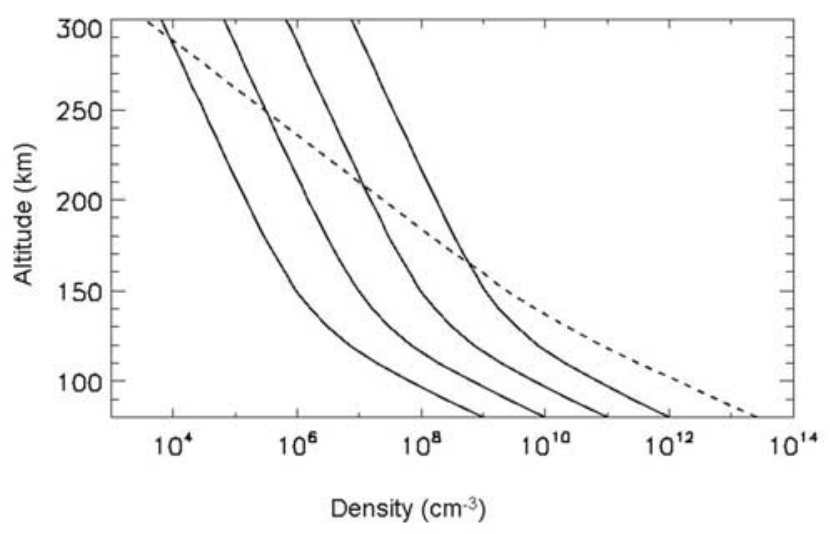

Figure 4. Four oxygen density profiles corresponding to densities at $80 \mathrm{~km}$ equal to $1 \times 10^{9} \mathrm{~cm}^{-3}, 1 \times 10^{10} \mathrm{~cm}^{-3}, 1 \times$ $10^{11} \mathrm{~cm}^{-3}$, and $1 \times 10^{12} \mathrm{~cm}^{-3}$, from left to right, respectively. The dashed line corresponds to the $\mathrm{CO}_{2}$ density of the models.

$4.44 \times 10^{17}$, and $s=0.5$ when $D_{O}$ is expressed in $\mathrm{cm}^{2} \mathrm{~s}^{-1}$ [Banks and Kockarts, 1973]. According to Krasnopolsky [2002], the weak escape of oxygen at the exobase does not affect the density profile, so we neglect oxygen escape when calculating our thermospheric oxygen profiles.

[8] Above the exobase at $200 \mathrm{~km}$, we use a one-dimensional (1-D) Chamberlain approach (without satellite particles) to describe the oxygen exospheric density. For this model, the oxygen density above $200 \mathrm{~km}$ depends only on the oxygen density and temperature at $200 \mathrm{~km}$ [Chamberlain, 1963]. We neglect any hot exospheric $\mathrm{O}$ components above the exobase, and we will only describe the effect of the core of the line on the intensity. Some possible effects of an oxygen hot population are given in section 4 . As a consequence, the $\mathrm{O}$ density profile from 80 to $1000 \mathrm{~km}$ is fully determined knowing the oxygen density at a given altitude. The reference altitude for the density is chosen to be $80 \mathrm{~km}$. Ten densities values at $80 \mathrm{~km}$ are used, varying from $1 \times 10^{9}$ to $1 \times 10^{12} \mathrm{~cm}^{-3}$, which encompass the range of densities $\left(\sim 10^{10}-10^{11} \mathrm{~cm}^{-3}\right)$ estimated from current photochemical models [Krasnopolsky, 2002; Fox, 2003]. Figure 4 displays four oxygen density profiles from 80 to $300 \mathrm{~km}$, corresponding to densities $1 \times$ $10^{9}, 1 \times 10^{10}, 1 \times 10^{11}$, and $1 \times 10^{12} \mathrm{~cm}^{-3}$ at $80 \mathrm{~km}$. The exobase density at $200 \mathrm{~km}$ varies as a consequence, from $\sim 1.5 \times 10^{5}$ to $1.3 \times 10^{8} \mathrm{~cm}^{-3}$. The slight deviation of the proportionality between the density range at the exobase and the density range at $80 \mathrm{~km}$ comes from the fact that the hydrostatic and diffusive equations are not independent, since oxygen is a major constituent. The sensitivity of the density profiles with respect to the exobase temperature and eddy diffusion is studied in section 4.2. In the following, we will use either $n_{\text {exo }}$ (density at the exobase) or $n_{80}$ (density at $80 \mathrm{~km}$ ) to define the model.

\subsection{Excitation of the $O$ I 130-nm Emission}

[9] The 130-nm triplet $(130.217,130.486$, and $130.603 \mathrm{~nm})$ emission is produced from the radiative decay of the $3 \mathrm{~s}^{3} \mathrm{~S}$ state to the ground $2 \mathrm{p}{ }^{3} \mathrm{P}$ state [Strickland and Donahue, 1970]. In this study, the two main sources of excitation of the $3 \mathrm{~s}{ }^{3} \mathrm{~S}$ states from the ground state are considered. These two sources of excitation are the resonant scattering of solar $130-\mathrm{nm}$ photons and the photoelectron impact on O [Strickland et al., 1972, 1973; Stewart et al., 1992]. The radiative transfer calculation (described in Appendixes A and B) is performed for each source separately, and the sum of the two solutions is used to estimate the total emergent intensity that should have been observed by SPICAM-UVS. The emergent intensity due to the solar photons depends on the solar flux at $130 \mathrm{~nm}$ in each line of the triplet $(130.2,130.4$, and $130.6 \mathrm{~nm})$. The total flux of the solar lines is derived from the measurement of the Solar Spectral Irradiance (SSI) provided by the Solar Radiation and Climate Experiment (SORCE) database [Rottman et al., 2006]. We have scaled this flux to the Martian position and taken into account the phase angle Earth-SunMars. The SORCE database provides the brightness measured between 130 and $131 \mathrm{~nm}$. We assume that all the solar brightness measured is due to the solar O 130-nm lines. The estimate of the relative solar flux in each line and of the brightness at the center of each line is done by adopting the solar line shapes derived from the Solar Maximum Mission and the Orbiting Solar Observatory 8 for the time period 1975-1985 [Gladstone, 1992]. Each solar line at Mars is then approximated by a flat "boxcar" line shape of 5 Doppler units' width, $5 \Delta \nu_{D}\left(T_{\text {exo }}\right)$, where $T_{\text {exo }}$ is the exobase temperature.

[10] Figure 5 displays the expected shapes and relative magnitude of each solar line. The flat shapes of the efficient solar lines assumed in our model are also displayed.

[11] As in previous studies [Strickland et al., 1972, 1973; Stewart et al., 1992], we do not consider the overlapping of the oxygen triplet by the $\mathrm{CO}$ fourth bands. The effect of this overlapping will be the subject of a forthcoming paper and is efficient essentially below $120 \mathrm{~km}$ [Barthelemy et al., 2008].

[12] A spherical, 3-D Monte Carlo, resonance line radiative transfer model has been used to compute the volume emission rate due to multiple scattering. This model is derived from an approach developed for the description of the heliospheric Lyman- $\alpha$ line [Quémerais, 2000] and has been updated to describe the oxygen triplet emissions in a planetary environment (Appendix A). The volume emission rate $S(\mu, z)$ due to solar photon scattering is computed on a discrete grid in solar zenith angle $(\mu)$ and altitude $(z)$ in a spherical model, and the multiple scattering effects are taken into account.

[13] The volume production rates due to photoelectron impact $S^{p e}{ }_{0}$ may be written as

$$
S_{0}^{p e}=n(z) g_{p e}[N(\mu, z)]
$$

where $n(z)$ is the atomic oxygen density, $g_{p e}$ is the electron impact frequency, and $N(\mu, z)$ is the slant column density of the atmosphere in the direction of the Sun. According to Stewart et al. [1992], the details of the equilibrium photoelectron fluxes are almost fully determined by the photoionization and electron collision cross sections of $\mathrm{CO}_{2}$ and are therefore insensitive to the abundance of $\mathrm{O}$. Thus, $N(\mu, z)$ is equal to the $\mathrm{CO}_{2}$ slant column density. The variation of $g_{p e}$ with $N$ is based on the work of Stewart [1970] in the terrestrial case. This primary volume production rate due to electron impact excitation of $\mathrm{O}$ is included in the radiative transfer model. The details of this computation are described in Appendix B. 

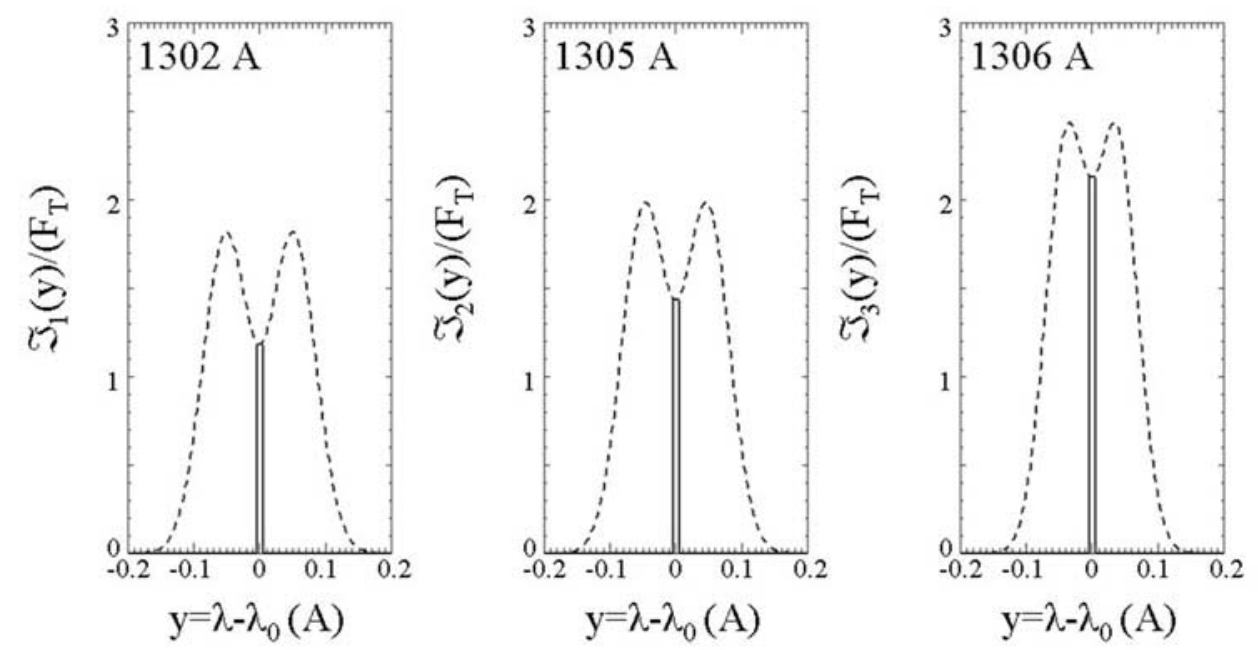

Figure 5. Theoretical shapes of the solar lines of the oxygen 130-nm triplet, a function of the wavelength $\lambda-\lambda_{0}$ in angstrom, where $\lambda_{0}$ is the center of each line. The sum of two Gaussians is taken from the work of Gladstone [1992]. The flat lines (thick solid lines at the center of each graph) are the approximate lines used in our model. The width of the line corresponds to 5 times the Doppler width for the exospheric temperature (here equal to $300 \mathrm{~K}$ ). $F_{1}, F_{2}$, and $F_{3}$ are the integrated flux of each line.

[14] The variation of the volume emission rates due to the solar photons and photoelectron impacts as a function of the altitude at $\mathrm{SZA}=30^{\circ}$ is displayed in Figure 6. In Figure 6 we display the primary emission rates and the emission rates due to multiple scattering. These profiles are calculated using a model of atmosphere defined by $T_{\text {exo }}=200 \mathrm{~K}$ and $n_{\text {exo }}=$ $1.5 \times 10^{7} \mathrm{~cm}^{-3}$ for the solar conditions of the group 3 observations. For this model, the effect of the multiple scattering is important below $400 \mathrm{~km}$. The order of magnitude of the primary emission rate due to photoelectrons is in good agreement with the emission rate profile $\left(\mathrm{SZA} \sim 48^{\circ}\right.$ ) given by Shematovich et al. [2008]. Compared to these authors, it seems that we overestimate it by a factor of 3 . Because we describe the photoelectron impact in a very simple way, a sensitivity analysis of the emission intensity profiles with respect to the $g_{p e}(0)$ parameter is presented in section 4.2.

\section{Data Analysis}

\subsection{Estimate of the Oxygen Density at the Exobase}

[15] To retrieve the oxygen density at the exobase, we use a least squares procedure that minimizes the $\chi^{2}$ function defined by

$$
\chi^{2}=\frac{1}{n-1} \sum_{i=1}^{i=n} \frac{\left[I_{o b s, i}-I_{\bmod , i}\left(n_{80}\right)\right]^{2}}{\sigma_{i}^{2}}
$$

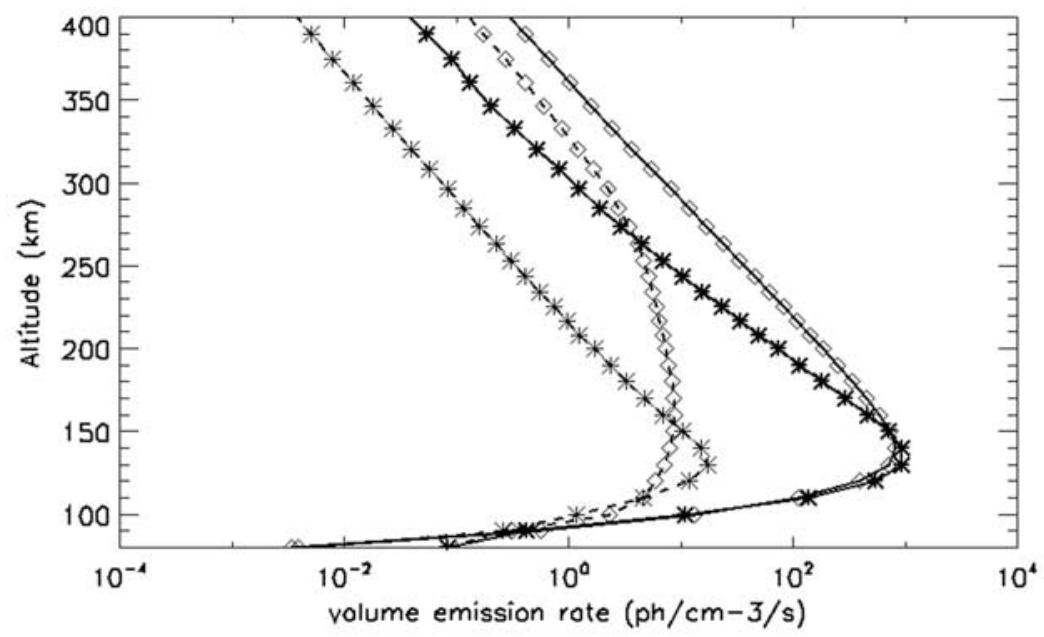

Figure 6. Volume emission rate profiles of oxygen excitation of the 130.4-nm lines due to solar photons (squares) and photoelectron impacts (asterisks). The dashed lines correspond to the primary emission rates, and the solid lines correspond to the multiple scattering emission rates. These profiles have been obtained for a model with an oxygen density at $80 \mathrm{~km}$ equal to $1 \times 10^{11} \mathrm{~cm}^{-3}$ and an exobase temperature equal to $200 \mathrm{~K}$ (see Figure 4) and for the solar conditions of group 3. 
Table 2. Oxygen Density at 80 and $200 \mathrm{~km}$ Derived From Each Group $^{\mathrm{a}}$

\begin{tabular}{ccc}
\hline Group & $n_{80} \times 10^{10}\left(\mathrm{~cm}^{-3}\right)$ & $n_{\text {exo }} \times 10^{6}\left(\mathrm{~cm}^{-3}\right)$ \\
\hline $1 \mathrm{a}$ & 5.8 & 8.7 \\
$1 \mathrm{~b}$ & 2.7 & 4.0 \\
$2 \mathrm{a}$ & 7.5 & 11.3 \\
$2 \mathrm{~b}$ & 5.8 & 8.7 \\
3a & 9.2 & 13.9 \\
3b & 3.6 & 5.4 \\
$4 \mathrm{a}$ & 9.5 & 14.2 \\
$4 \mathrm{~b}$ & 3.1 & 4.6 \\
$5 \mathrm{a}$ & 8.6 & 13.0 \\
\hline
\end{tabular}

${ }^{\text {a }}$ The exobase altitude is $200 \mathrm{~km}$.

where $n$ is the number of points in the observed intensity profile, $I_{o b s, i}$ is the observed intensity for the $i$ th line of sight of the fitted profile, $I_{\text {mod, } i}\left(n_{80}\right)$ is the modeled intensity for the same line of sight assuming an oxygen density $n_{\text {exo }}$ at $80 \mathrm{~km}$, and $\sigma_{i}$ is the standard deviation of the individual sample (see Figure 3). Table 2 displays the density at $80 \mathrm{~km}$ and at the exobase, which minimizes the $\chi^{2}$ function of each group of observations. The average density of all $80-\mathrm{km}$ points is $6.2 \times 10^{10} \mathrm{~cm}^{-3}$, which is in good agreement with the photochemical model of Krasnopolsky [2002] and previous observations. This average density corresponds to an exobase density of $9.3 \times 10^{6} \mathrm{~cm}^{-3}$.

[16] The 80-km and exobase oxygen densities are systematically lower at high SZA (groups 1b, 2b, 3b, and 4b) than at low SZA (groups 1a, 2a, 3a, 4a, and 5a). The average density at the exobase is $1.2 \times 10^{7} \mathrm{~cm}^{-3}$ at SZA $\sim 30^{\circ}$ and 2 times smaller at high SZA $\left(5.7 \times 10^{6} \mathrm{~cm}^{-3}\right)$.

[17] Figure 7 displays an example of the fit for group $3 \mathrm{a}$. The theoretical contributions of the solar photons and photoelectrons are also shown. At $200 \mathrm{~km}$, the contribution from photoelectron excitation $(\sim 80 \mathrm{R})$ is $\sim 20 \%$ of the total intensity (430 R), which is in good agreement with the values between 16 and 25\% deduced by Stewart et al. [1992] from Mariner data.

[18] The best profile displayed in Figure 7 does not fit the data very well. However, considering the assumptions (analytical description of the photoelectron impact excitation, Maxwellian velocity distribution functions in the exosphere, spherical symmetry of the density, and no hot $\mathrm{O}$ population) and the badly constrained parameters (exospheric temperature, electron impact excitation frequency, and eddy mixing coefficient) used in the model, the result seems reasonable. The uncertainties on the model, indicated in Figure 7 (dotted lines), are defined in section 4.2. Moreover, this result is well supported by photochemical models [Krasnopolsky, 2002]. In section 4.2 , in order to estimate the uncertainties due to the model on the values of the oxygen density at the exobase, we test the sensitivity of our results to different constrained parameters.

\subsection{Uncertainty in the Oxygen Density at the Exobase}

[19] As mentioned, the derived oxygen density values of section 4.1 are dependent on poorly constrained parameters, such as eddy mixing coefficient $K(z)$, absolute calibration (or solar flux) described by a multiplicative factor $A$, photoelectron impact frequency parameter $g_{p e}(0)$, and exobase temperature $T_{\text {exo }}$. Other parameters such as Gauss/Voigt line profiles used in the Monte Carlo radiative transfer model, nonspherical symmetry of the oxygen density (i.e., variations with latitude or longitude in the vertical $O$ density profile), or departure of the exospheric oxygen density profile from the Chamberlain's approximation will be studied in a future work. Using a Voigt line profile should increase partly the intensity of the models at low altitudes, but the uncertainty induced by a Gauss profile with respect to a Voigt profile is expected to be smaller than other parameters listed above.

[20] In sections 4.2.1-4.2.4 the uncertainty in the oxygen density derived from the uncertainty in a given parameter is studied by varying the value of each parameter while keeping the others fixed at the values determined for them in section 4.1. The nominal values of the parameters listed above are, respectively, $K(z)=1.2 \times 10^{12} \times\left[T_{\text {exo }} / n(z)\right]^{1 / 2}$ [Krasnopolsky, 2002], $A=1, g_{p e}(0)=1.7 \times 10^{-7} \mathrm{~s}^{-1} \times$ $\left[F_{10.7} / 54\right]$, and $T_{\text {exo }}=200 \mathrm{~K}$. We present here the results for the observations belonging to groups $3 \mathrm{a}$ and $3 \mathrm{~b}$ (low and high SZA). However, the conclusions of this analysis have been found valid for the whole set of observations presented in Table 1.

\subsubsection{Sensitivity to the Eddy Diffusion Coefficient}

[21] In order to test the sensitivity of the intensity to the eddy mixing coefficient, we use models with the same oxygen density at (and above) the exobase $\left(1.5 \times 10^{7} \mathrm{~cm}^{-3}\right.$ at the exobase) but with different mixing diffusion coefficients (therefore different oxygen thermospheric density). The density profiles displayed in Figure 8 a have been obtained using eddy mixing diffusion coefficients $K(z)=0.1 \times K_{0}(z)$, $1 \times K_{0}(z)$, and $10 \times K_{0}(z)$, where $K_{0}(z)$ is the nominal eddy mixing coefficient. As expected, when this coefficient

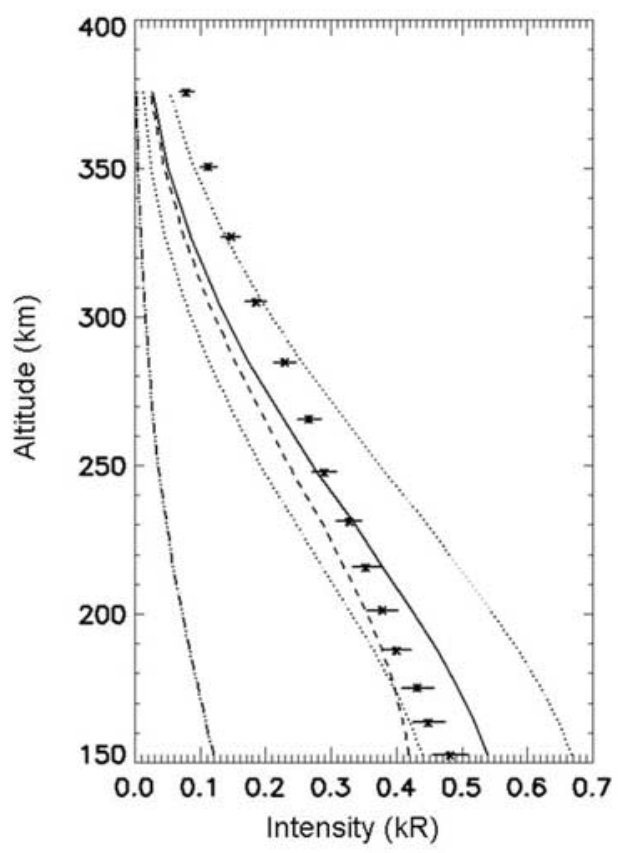

Figure 7. Example of fit (solid line) of the measured emission intensity. The black points represent the data, and the horizontal lines represent the standard deviation. The dashed line represents the contribution of the solar source $(\sim 80 \%)$. The dash-dotted line represents the contribution of the photoelectron impact source $(\sim 20 \%)$. The dotted lines represent the intensity profiles corresponding to upper and lower uncertainties on the exobase density. 

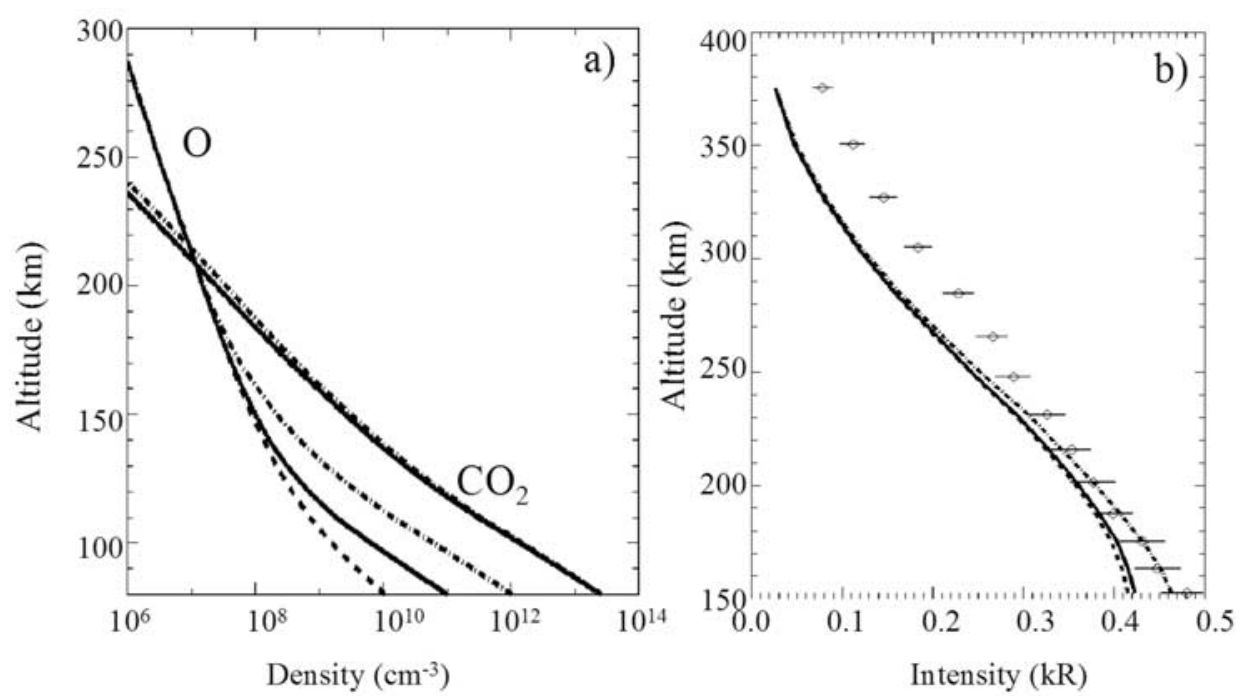

Figure 8. Sensitivity of the integrated emission intensity with respect to the eddy diffusion coefficient. (a) Different models with the same exospheric density (exobase density equal to $1.5 \times 10^{7} \mathrm{~cm}^{-3}$ and exobase temperature equal to $200 \mathrm{~K}$ ) but with different thermospheric profiles corresponding to a mixing diffusion coefficient 10 times lower than the model described in section 4.1 (dashed line), equal to the model (solid line), and 10 times higher than the model (dash-dotted line), respectively. (b) Intensity profiles deduced from each model with the same legend as well as the observed profile (group 3a).

increases, the altitude of the homopause increases. When eddy diffusion dominates, then $\mathrm{O}$ and $\mathrm{CO}_{2}$ have the same scale heights; when molecular diffusion dominates, each species has its own scale height (Figure 8a). Figure $8 \mathrm{~b}$ shows the intensity profiles derived from these three models for group 3 at low SZA. The calculated intensity is not very sensitive to the eddy mixing coefficient; between the two extreme models, the intensity at $150 \mathrm{~km}$ only varies by $<10 \%$, while the oxygen density at $80 \mathrm{~km}$ varies 2 orders of magnitude.

[22] In order to determine the altitude range probed by SPICAM-UVS when measuring the O 130.4-nm emission, the line of sight has been divided into 50 sections of equal length $(\sim 70 \mathrm{~km})$. Figures $9 \mathrm{a}$ and $9 \mathrm{~b}$ display the contribution of each of these sections (in percent of the total intensity) with respect to its altitude (which is taken as the altitude of the center of each section). Figure 9a displays the result for the solar source, and Figure 9b displays the result for the photoelectron source. The line of sight considered in Figure 9 corresponds to a line of sight of group 3 with a tangential point near $150 \mathrm{~km}$. The three models have densities at $80 \mathrm{~km}$ equal to $1 \times 10^{9}, 1 \times 10^{11} \mathrm{~cm}^{-3}$, and $1 \times 10^{12} \mathrm{~cm}^{-3}$, respectively. When the simulated density increases, the optical thickness increases. As a consequence, the expected region probed by SPICAM-UVS moves toward higher altitudes. For these models, the altitude range really seen by SPICAM-UVS varies from $\sim 170$ to $\sim 340 \mathrm{~km}$. Therefore SPICAM-UVS probes essentially the exosphere and in a minor way the thermosphere in the cases considered in this paper.

\subsubsection{Sensitivity to the Absolute Calibration}

[23] Another source of uncertainty is related to the absolute calibration of SPICAM-UVS. Such uncertainty derives from the uncertainty in the efficiency area (that is, on the calibration based on already calibrated stars), estimated as being of the order of $15 \%$ [Bertaux et al., 2006], or on the method used to retrieve the total intensity [Leblanc et al., 2006]. Another source of uncertainty, which has the same effect as the calibration uncertainty, is related to the uncertainty in the solar flux given in the SORCE database and used to calculate the oxygen emission intensity in our model. To take into account such kind of uncertainties, we introduce a factor $A$ to the intensity derived from SPICAM-UVS. The variations of the derived oxygen density at $80 \mathrm{~km}$ with respect to the value of this factor $A$, chosen as varying between 0.7 and 1.3 , are given in Table 3.

[24] As expected for an optically thick emission, the derived densities at $80 \mathrm{~km}$ are very sensitive to the calibration and vary nonlinearly with the absolute intensity. For each observation the $\chi^{2}$ values are decreasing when the multiplicative factor is increasing. This may suggest that the nominal calibration underestimates rather than overestimates the real intensity. An uncertainty of $20 \%$ in the absolute intensity implies an uncertainty in the oxygen density retrieved at $80 \mathrm{~km}$ of $\sim 70 \%$.

\subsubsection{Sensitivity to the Photoelectron Impact \\ Frequency}

[25] A third source of uncertainty in the oxygen density may be due to the description of the photoelectron impact $g_{p e}(0)$ in the model. An overestimation of $g_{p e}(0)$ by a factor of 4 is a reasonable upper uncertainty because it corresponds to the ratio between the cross sections used by Stewart et al. [1992] and those by Strickland et al. [1973]; this overestimation also encompasses the uncertainties of the O 130-nm electron impact excitation cross section given by Barklem [2007]. In another way, we considered an underestimation by a factor of 4 . The variation of the inferred density at $80 \mathrm{~km}$ with respect to different values of the photoelectron impact excitation frequency $g_{p e}(0)$ is given in Table 4 . 
a)

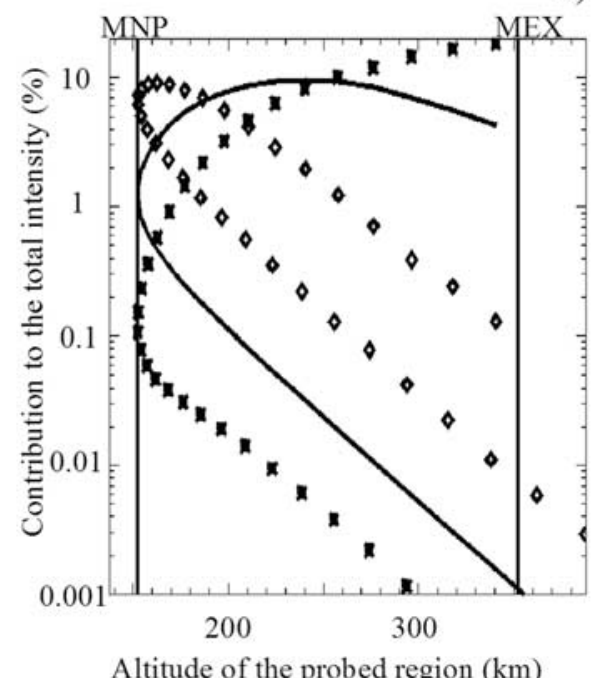

b)

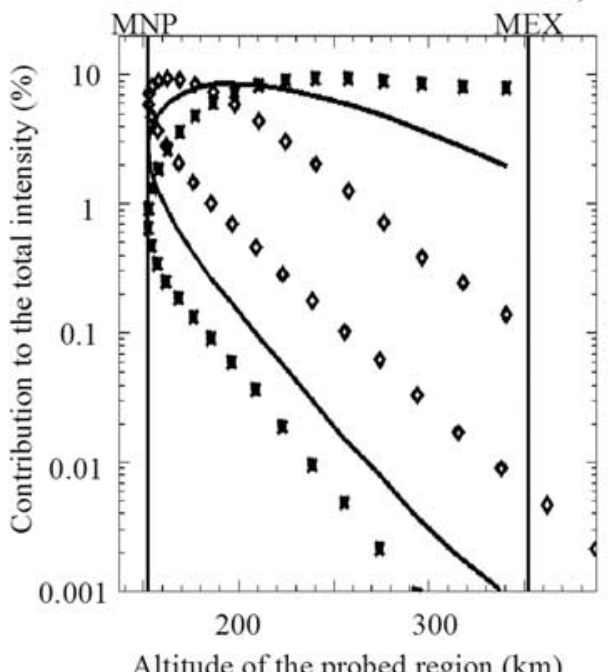

Figure 9. Contribution of different sections of the line of sight to the total integrated intensity with respect to the altitude of the section (taken as the altitude of the center of each section). The different lines correspond to the different models with densities at $80 \mathrm{~km}$ equal to $1 \times 10^{9} \mathrm{~cm}^{-3}$ (diamonds), $1 \times$ $10^{11} \mathrm{~cm}^{-3}$ (solid lines), and $1 \times 10^{12} \mathrm{~cm}^{-3}$ (asterisks). (a) The results for the solar source, and (b) The results for the photoelectron impact source. The two vertical lines represent the altitude of Mars Express (MEX) and the altitude of the tangent point of the line of sight (Mars nearest point, or MNP; here near $150 \mathrm{~km})$.

[26] The inferred density at $80 \mathrm{~km}$ decreases when the photoelectron frequency increases, because of the increase of the total intensity due to the excitation by the photoelectron. The lower uncertainty in the oxygen density is equal to $155 \%$ at low SZA and $100 \%$ at high SZA, and the upper uncertainty is equal to $\sim 70 \%$ at low SZA and $\sim 40 \%$ at high SZA. A description of the photoelectron impact with a more accurate model describing the photoelectron transport as used by Stewart et al. [1992] is therefore highly needed to reduce the uncertainty due to this parameter.

Table 3. Variations of the Oxygen Density at the Exobase, $200 \mathrm{~km}$, Derived From Each Group of Observations When an Uncertainty in the Absolute Calibration is Introduced ${ }^{\mathrm{a}}$

\begin{tabular}{lcc}
\hline$A$ & $n_{\text {exo }} \times 10^{6}\left(\mathrm{~cm}^{-3}\right)$ \\
\hline & Low SZA & \\
0.7 & & 5.5 \\
0.8 & & 8.0 \\
0.9 & & 10.5 \\
1.0 & & 13.9 \\
1.1 & 18.1 \\
1.2 & & 22.4 \\
1.3 & & 28.0 \\
& & \\
0.7 & & \\
0.8 & & 2.1 \\
0.9 & & 3.0 \\
1.0 & & 4.0 \\
1.1 & & 5.4 \\
1.2 & & 7.1 \\
1.3 & & 9.0 \\
\hline
\end{tabular}

${ }^{\mathrm{a}}$ The $A$ coefficient represents a multiplicative factor of the intensity observed by SPICAM/MEX.

\subsubsection{Sensitivity to the Exobase Temperature}

[27] The modeled temperature is fully defined by the temperature at the exobase [Krasnopolsky, 2002]. In particular, the exosphere is assumed to be isothermal. The temperature is an important parameter because it defines the scale height of the oxygen in the thermosphere as well as in the exosphere. Moreover, it increases the width of the Doppler line in the radiative transfer model. We choose exospheric temperatures varying between 175 and $400 \mathrm{~K}$ in agreement with the exospheric temperatures estimated from the MTGCM model [Bougher et al., 1999]. Figure 10 displays the best fits for each exospheric temperature. Above $250 \mathrm{~km}$ the calculated intensity increases with increasing exobase temperature. Inversely, the oxygen density at the exobase derived from the study will decrease with increasing exobase temperature as shown in Table 5.

Table 4. Variations of the Oxygen Density at the Exobase, $200 \mathrm{~km}$, Derived From Group 3 Observations With the Photoelectron Frequency $g_{p e}(0)$

\begin{tabular}{ccc}
\hline$g_{p e}(0) / g_{p e}($ Standard $)$ & $n_{\text {exo }} \times 10^{6}\left(\mathrm{~cm}^{-3}\right)$ \\
\hline & Low SZA & \\
$1 / 4$ & & 23.5 \\
$1 / 2$ & & 19.0 \\
1 & & 13.9 \\
4 & & 9.3 \\
& & 5.8 \\
& & \\
$1 / 4$ & High SZA & \\
$1 / 2$ & & 7.8 \\
1 & & 6.8 \\
2 & & 5.4 \\
4 & & 3.9 \\
\hline
\end{tabular}




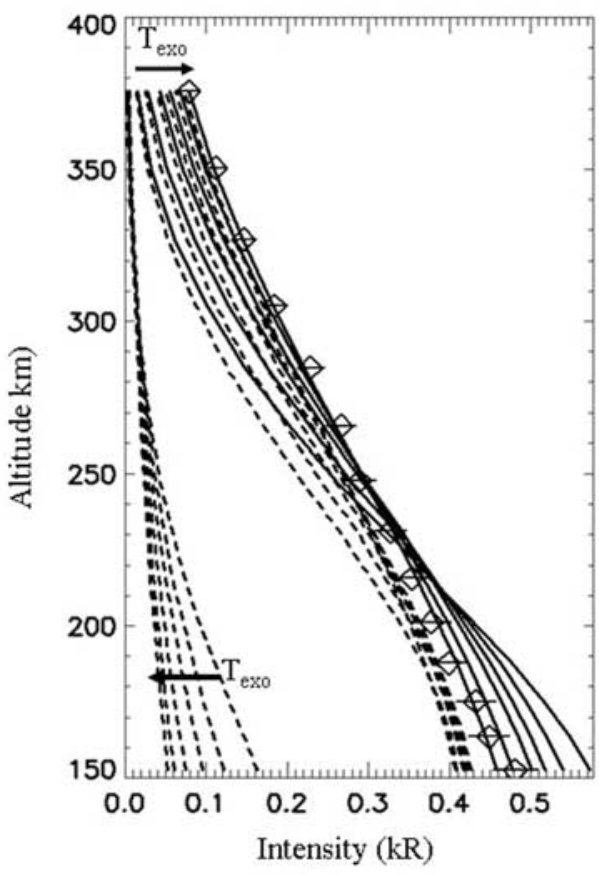

Figure 10. Best fit obtained for different exobase temperatures (from 175 to $300 \mathrm{~K}$ with a step of $25 \mathrm{~K}$ ). The dashed lines represent the contribution of the photoelectron impact, the dashed lines on the left represent the contribution of the photoelectron impact, the dashed lines on the right represent the contribution of the solar photons, and the solid lines represent the total intensity. The models with a high exobase temperature provide the best fit to the observed intensity profiles.

[28] The best fits are obtained when the exobase temperature is around $300 \mathrm{~K}$. For the same orbits, the analysis of the $\mathrm{CO}_{2}^{+}$bands and the $\mathrm{N}_{2}$ Vegard-Kaplan bands below $200 \mathrm{~km}$ suggests a temperature between 175 and $225 \mathrm{~K}$ [Leblanc et al., 2007]. The uncertainty in the oxygen density at $80 \mathrm{~km}$ due to the exobase temperature can be estimated to be equal to $\sim 50 \%$. The uncertainty in the oxygen at the exobase is smaller and equal to $\sim 25 \%$. The effect of a hot population produced by $\mathrm{O}_{2}^{+}$recombination [Nagy and Cravens, 1988] could explain the fact that the profiles are better reproduced with temperatures larger than $200 \mathrm{~K}$. According to a recent model [Chaufray et al., 2007], the hot population could be a dominant population only above $\sim 550 \mathrm{~km}$, but the extended wings of the line created by this hot population could produce a low uniform intensity background whose relative contribution would increase with altitude. In section 4.3 we try to estimate the brightness due to the hot population.

\subsection{Effect of a Hot Population}

[29] To estimate the effect of a hot population on the observed brightness, we have computed a first-order calculation. The density profile used to describe the hot population is the one computed by Chaufray et al. [2007] for minimum solar conditions, extrapolated exponentially below $550 \mathrm{~km}$ until $200 \mathrm{~km}$. The densities of the cold and hot oxygen populations are displayed in Figure 11. The temperature of the hot population is assumed to be uniform and equal to
$6000 \mathrm{~K}$, which corresponds to an average energy of $\sim 0.6 \mathrm{eV}$ by atoms and is near the values found by Krestyanikova and Shematovich [2005] and Cipriani et al. [2007].

[30] The volume emission rates are computed assuming that the atmosphere is optically thin. In this case, the volume emission rate at the line $i$ is proportional to the density. The volume emission rate is then equal to the density multiplied by the product of the percentage of oxygen in the ground state susceptible to absorbing the photons emitted in the line $i: p_{i}$ (see Appendix A for more details) and the excitation parameter $g_{\text {exc }}$ given by the convolution of the solar lines profile and the Gaussian absorption profile:

$$
g_{\text {exc }}=\sum_{j=1}^{j=3} p_{j} \sigma_{0, j} \int_{-\infty}^{+\infty}\left(\pi F_{\lambda^{\prime}, j}\right) \exp \left[-\left(\lambda^{\prime}-\lambda_{0, j}\right)^{2} / \Delta \lambda_{D, j}^{2}\right] d \lambda^{\prime},
$$

where $\sigma_{0, j}$ is the absorption cross section at the center of the $j$ th line, $\pi F_{\lambda j} j$ is the spectral solar flux of the line $j, \lambda_{0, j}$ is the wavelength at the center of the line $j$, and $\Delta \lambda_{D, j}$ is the Doppler width of the line $j$. Because here we assume a uniform temperature for the hot population, $p_{j}, \sigma_{0, j}$, and $\Delta \lambda_{D, j}$ are also uniform. Using the analytical profile lines given by Gladstone [1992] and displayed in Figure 5, the calculation of equation (4) is straightforward, and we find an excitation factor $g_{\text {exc }}$ equal to $1.35 \times 10^{-5} \mathrm{~s}^{-1}$.

[31] We take into account the auto-absorption when we calculate the integrated intensity along the line of sight. The integrated intensity obtained for group $3 \mathrm{a}$ is equal to $\sim 11 \mathrm{R}$ between 200 and $400 \mathrm{~km}$. When we use a hot oxygen temperature of $10,000 \mathrm{~K}$, the integrated intensity is equal to $\sim 12 \mathrm{R}$. Therefore a hot population can partly explain the differences between the best model obtained in section 4.1.

Table 5. Variations of the Density at 80 and $200 \mathrm{~km}$ Derived From Group 3 Observations When the Exobase Temperature is Varied ${ }^{\mathrm{a}}$

\begin{tabular}{lcc}
\hline$T_{\text {exo }}(\mathrm{K})$ & $n_{80} \times 10^{10}\left(\mathrm{~cm}^{-3}\right)$ & $n_{\text {exo }} \times 10^{6}\left(\mathrm{~cm}^{-3}\right)$ \\
175 & Group $3 a$ & \\
200 & 13.4 & 16.5 \\
225 & 9.2 & 13.9 \\
250 & 6.5 & 11.0 \\
275 & 4.8 & 8.9 \\
300 & 3.7 & 7.3 \\
325 & 3.0 & 6.0 \\
350 & 2.4 & 4.9 \\
375 & 2.0 & 4.2 \\
400 & 1.7 & 3.6 \\
& 1.5 & 3.1 \\
& & \\
175 & Group $3 b$ & 6.6 \\
200 & 5.3 & 5.4 \\
225 & 3.6 & 4.3 \\
250 & 2.5 & 3.6 \\
275 & 2.0 & 2.9 \\
300 & 1.5 & 2.3 \\
325 & 1.2 & 2.0 \\
350 & 1.0 & 1.7 \\
375 & 0.8 & 1.5 \\
400 & 0.7 & 1.3 \\
\hline
\end{tabular}

${ }^{\mathrm{a}}$ For all groups of observations, the assumption of a high exospheric temperature $(300 \mathrm{~K})$ provides the best fit. 


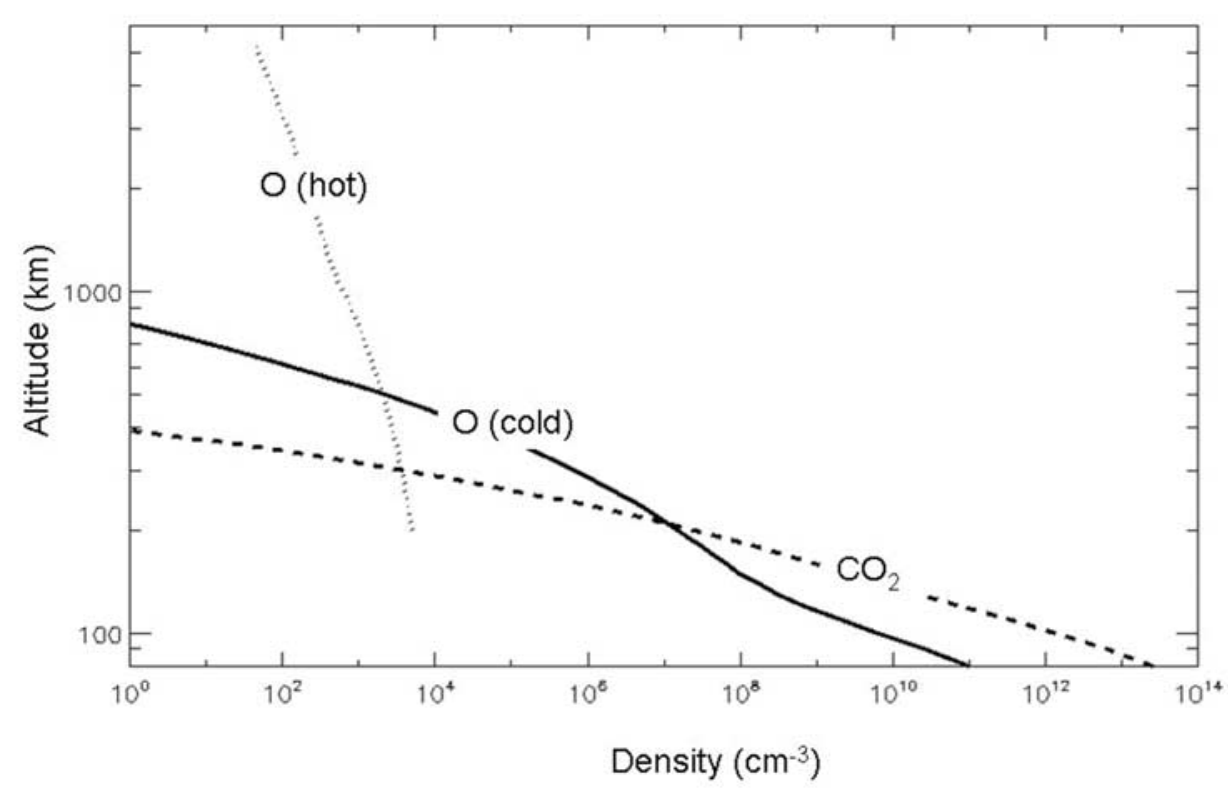

Figure 11. Density profiles of $\mathrm{CO}_{2}, \mathrm{O}$ (cold), and $\mathrm{O}$ (hot) used to estimate the contribution of the hot oxygen component to the intensity.

A hot oxygen density that is denser (by a factor of $\sim 5$ ) than that modeled by Chaufray et al. [2007] could improve the fit.

\section{Discussion}

\subsection{Exospheric Density}

[32] From the sensitivity study of section 4.2, the total uncertainty in the oxygen density as derived from the observations is equal to a factor of 2 or 3 on the nominal values given in section 4.1. Therefore the oxygen exobase density derived from our analysis is between 0.7 and $2.4 \times 10^{7} \mathrm{~cm}^{-3}$ at low SZA and between 0.3 and $1.0 \times 10^{7} \mathrm{~cm}^{-3}$ at high SZA. Actually, the different parameters considered in section 4.2 do not act independently, and therefore this uncertainty is probably overestimated. The ratio between the densities at low SZA and those at high SZA should be less dependent on these parameters. As an example, an uncertainty in the calibration implies the same correction to the data at low and high SZA and, as a consequence, a significantly smaller uncertainty in the divided exobase densities (Table 3).

[33] Recently, Mazarico et al. [2007] have estimated the total density of the Martian atmosphere at $\sim 400 \mathrm{~km}$ altitude by using radio-tracking data and precise orbit determination on the Mars Odyssey spacecraft between March 2002 and November 2005. These authors found a density between $10^{-15}$ and $10^{-14} \mathrm{~kg} \mathrm{~m}^{-3}$ and a scale height between 25 and $45 \mathrm{~km}$. The main species contributing to the mass density at $400 \mathrm{~km}$ are $\mathrm{O}, \mathrm{H}, \mathrm{H}_{2}$, and $\mathrm{He}$. (The extrapolations of the $\mathrm{CO}$ and $\mathrm{N}_{2}$ densities from Krasnopolsky [2002] show that they are minor contributors, even in terms of mass density.)

[34] The mass density $\rho$ of the exosphere is

$$
\rho=\sum_{i} \rho_{i}
$$

where $\rho_{i}=n_{i} m_{i}$ is the mass density of species $i$. The atmospheric scale height can be linked to the scale height of the different species. If we assume a simple exponential law to describe the local density profile near $400 \mathrm{~km}$ as used by Mazarico et al. [2007], we find

$$
\frac{1}{H_{\rho}}=\frac{1}{\rho}\left(\sum_{i} \frac{\rho_{i}}{H_{i}}\right),
$$

where $H_{\rho}$ is the scale height of the total mass density and $H_{i}$ is the scale height of species $i$.

[35] The hydrogen density is estimated from SPICAM above the south pole [Chaufray et al., 2008], which corresponds to the observations analyzed by Mazarico et al. [2007]. The $\mathrm{H}_{2}$ and He densities are estimated by extrapolating the exobase densities given by Krasnopolsky [2002] with a simple Chamberlain approach, for an exospheric temperature equal to $200 \mathrm{~K}$. This author found an exobase hydrogen density in reasonable agreement with SPICAM. We consider the oxygen density estimated in this paper at $400 \mathrm{~km}$ for SZA between $55^{\circ}$ and $90^{\circ}$.

[36] The densities at $400 \mathrm{~km}$ are therefore $1 \pm 1 \times 10^{5}$, $1 \pm 1 \times 10^{5}, 3 \pm 3 \times 10^{4}, 5 \pm 3 \times 10^{4} \mathrm{~cm}^{-3}$ for $\mathrm{H}, \mathrm{H}_{2}, \mathrm{He}$, and $\mathrm{O}$, respectively. Using these values, we find a mass density $\rho$ between 0.5 and $3.5 \times 10^{-15} \mathrm{~kg} \mathrm{~m}^{-3}$ in good agreement with the estimate of Mazarico et al. [2007]. Neglecting any hot populations and assuming a single temperature for all species, the mass density scale height $H_{\rho}$ of $35 \mathrm{~km}$ corresponds to an exospheric temperature between 140 and $240 \mathrm{~K}$, in good agreement with Leblanc et al. [2007].

[37] The presence of a hot oxygen population should not change this temperature derived from a scale height. Indeed, as shown in equation (6), the term $\rho_{\text {hot }} / H_{\text {hot }}$ should be small with respect to the other terms. Figure 7 shows that for the best model considered here, the intensity is lower than the observed intensity at high altitudes. This effect has also been observed on Earth by the Berkeley EUV airglow rocket spectrometer (BEARS) [Cotton et al., 1993]. Cotton et al. 
[1993] suggest that this discrepancy may be due to an emission induced by photons escaping the lower thermosphere and scattered in the large wings of the velocity distribution of a hot oxygen corona component. However, the investigation of the effect of the hot population on these EUV data has been shown to be insufficient [Hubert et al., 1999]. A full radiative transfer calculation including both cold and hot oxygen components and non-Maxwellian velocity distributions would be useful to investigate further the effect of the hot population.

\section{2. $[\mathrm{O}] /\left[\mathrm{CO}_{2}\right]$ Mixing Ratio}

[38] As shown in section 4.2, the emission intensity measured above $150 \mathrm{~km}$ by SPICAM-UVS is essentially constrained by the oxygen exospheric density profiles. The thermospheric oxygen density profile is therefore badly constrained by SPICAM-UVS observations. Despite this limitation, it is interesting to derive the value of the $[\mathrm{O}] /\left[\mathrm{CO}_{2}\right]$ mixing ratio using the oxygen profiles given in section 4.1. The average value of the mixing ratio $[\mathrm{O}] /\left[\mathrm{CO}_{2}\right]$ at $135 \mathrm{~km}$ is equal to $0.9 \%$. This value is slightly lower than the value $(\sim 1.25 \%)$ deduced from Viking ion profiles at SZA $\sim 60^{\circ}$ during low solar activity [Hanson et al., 1977]. Hanson et al. [1977] used a simple photochemical approach in order to reproduce the observed ion profiles. In another way, this value is slightly higher than the mixing ratio deduced by Stewart et al. [1992] from Mariner's missions (between 0.4 and $0.7 \%$ ) during high solar activity. At low SZA the average value of the mixing ratio is found to be around $1.2 \%$, and at high SZA this value is equal to $0.6 \%$ These values are in good agreement with the MTGCM model [Bougher et al., 1999] and cannot explain the low thermospheric temperature deduced by Keating et al. [1998] from the accelerometer's measurement of MGS. Keating et al. [1998] attributed this low thermospheric temperature to a possible higher $[\mathrm{O}] /\left[\mathrm{CO}_{2}\right]$ mixing ratio than that predicted or to the presence of gravity waves. Because the temperatures deduced by SPICAM are lower than those predicted by the MTGCM model [Leblanc et al., 2006, 2007], an uncertainty in the collision coefficient between $\mathrm{CO}_{2}$ and $\mathrm{O}$ and therefore on the cooling rate or in the solar EUV/UV heating efficiency could be another explanation of the observed discrepancy.

\section{Conclusion}

[39] We present here the first detailed analysis of the oxygen 130.4-nm line observed by SPICAM-UVS aboard Mars Express. The present coverage of measurements in terms of solar zenith angle, aerocentric longitude, and local time is limited because limb-viewing observations of the dayglow imply a particular orientation of the spacecraft. The measurements presented in this paper are the first of the oxygen in the upper atmosphere since Mariner's missions 30 years ago. Comparison between oxygen data and density models coupled to a radiative transfer approach leads to derivation of an oxygen density at the exobase equal to $1.2_{-0.5}^{+1.2} \times 10^{7} \mathrm{~cm}^{-3}$ at $\mathrm{SZA}<60^{\circ}$ and equal to $0.6_{-0.3}^{+0.4} \times 10^{7} \mathrm{~cm}^{-3}$ at $\mathrm{SZA}>60^{\circ}$. Because Mars Express is above the exobase, the observed oxygen emission is more sensitive to the exospheric oxygen than to the thermospheric oxygen. A simple diffusion model allows one to extrapolate the density below the exobase, leading to a $[\mathrm{O}] /\left[\mathrm{CO}_{2}\right]$ mixing ratio between 0.6 and $1.2 \%$, in good agreement with previous measurements [Hanson et al., 1977; Stewart et al., 1992]. Exospheric temperatures higher than $200 \mathrm{~K}$ provide the best fit to the data and could be due to the presence of a hot population produced by dissociative recombination of $\mathrm{O}_{2}^{+}$[Krestyanikova and Shematovich, $2005]$. In order to confirm this conclusion, a model of radiative transfer needs to be developed that takes into account a hot oxygen population and describes non-Maxwellian velocity distribution. Reducing the model's uncertainties by using a more realistic description of the photoelectron impact frequency is also needed to better constrain the oxygen density at the exobase. Such a model could also be useful to study the O $1356 \AA$ line. This line, identified on SPICAM-UVS spectra below $200 \mathrm{~km}$ in altitude [Leblanc et al., 2006], is an optically forbidden transition, and its intensity is weaker than the intensity of the 130.4-nm triplet. Finally, the CO fourth positive bands overlapping partly the $\mathrm{O}$ 130.4-nm emission could also contribute to the observed emission, in particular by redistributing part of the 130.4-nm emission intensity toward longer wavelengths [Barthelemy et al., 2008].

\section{Appendix A: A Radiative Transfer Code for the Solar Source}

[40] Because the O 130.4-nm triplet is optically thick, we use a spherical radiative transfer model that takes into account multiple scattering in calculating the volume emission rates. The optical thickness at the center of the first line $(130.217 \mathrm{~nm})$ for the SPICAM/MEX observations (for the nominal model) is $\sim 720$ at $150 \mathrm{~km}, 130$ at $200 \mathrm{~km}$, and 5 at $300 \mathrm{~km}$. Our radiative transfer model is derived from the Monte Carlo model developed by Quémerais [2000] to describe the Lyman- $\alpha$ brightness of the interplanetary medium. This Monte Carlo model takes into account temperature variations with altitude and partial frequency redistribution. For each oxygen density profile, twenty million solar photons are followed into the atmosphere until they escape or they are absorbed by $\mathrm{CO}_{2}$.

[41] Each test photon comes from a disk orthogonal to the Sun-Mars axis and whose radius is equal to $4400 \mathrm{~km}$ (Martian radius plus altitude upper limit). The position on this disk is determined randomly.

[42] In our model, the relation between the flux at the center of each line and the integrated flux of each line is deduced from the profiles of Gladstone [1992] and given by

$$
\frac{F_{i}(0)}{F_{i}}=\frac{1}{\sqrt{\pi} x_{0 i}}\left\{\exp \left[-\left(\frac{x_{1 i}}{x_{0 i}}\right)^{2}\right]\right\}
$$

where $F_{i}(0)$ is the solar flux at the center of the line $i, F_{i}$ is the integrated solar flux of the line $i$, and $x_{0 i}$ and $x_{1 i}$ are the parameters of each line estimated by Gladstone [1992]. The ratios between the integrated solar flux of the $i$ th line and the total flux of the three lines $f_{i}=F_{i} /\left(F_{1}+F_{2}+F_{3}\right)$ used in this study are also those estimated by Gladstone [1992].

[43] Because the temperature of the Martian atmosphere is very low compared to the temperature of formation of the solar lines, the solar line can be assumed to be flat in the spectral region studied here $\left[-2.5 \Delta \nu_{D, i}\left(T_{\text {exo }}\right) ; 2.5 \Delta \nu_{D, i}\left(T_{\text {exo }}\right)\right]$, where $\Delta \nu_{D, i}\left(T_{\text {exo }}\right)$ is the Doppler width of the line $i$ (which is assumed independent of the line hereinafter). Figure 5 displays 
the profile of each line normalized by $F_{1}+F_{2}+F_{3}$ and the flat profiles used in this study for an exospheric temperature equal to $300 \mathrm{~K}$. To determine the solar line of emission of each simulated solar photon, we use

$$
\begin{aligned}
& 0<r<\frac{G_{1}}{G} \text { : line1 } \\
& \frac{G_{1}}{G}<r<\frac{G_{1}+G_{2}}{G}: \text { line2 } \\
& \frac{G_{1}+G_{2}}{G}<r<1 \text { : line3 }
\end{aligned}
$$

In equation (A2), $r$ is a random number between 0 and 1 ; $G_{1}, G_{2}$, and $G_{3}$ are the integrated fluxes over the flat shape used in this study $\left(G_{i}=5 \times F_{i}(0) \times \Delta \nu_{D}\right)$ (thick solid lines of Figure 5); and $G=G_{1}+G_{2}+G_{3}$. The ratios $G_{i} / G$ are independent of the integrated solar flux; therefore each model can be scaled to any of the observations. The normalized frequency of the solar photon is given by

$$
x=5 r-2.5 .
$$

This implies that the frequency is given by

$$
\nu=\nu_{0, i}+\Delta \nu_{D, r e f}(5 r-2.5)
$$

where $\nu_{0, i}$ is the frequency at the center of the line $i$ and $\Delta \nu_{D, \text { ref }}$ is the Doppler width of the line for a reference temperature $T_{\text {ref }}$ (here the exospheric temperature).

[44] The direction of propagation of the solar photon is the Sun-Mars direction. We then determine the optical thickness that the solar photon needs to cross before its first scattering:

$$
\tau_{s}=-\log (r)
$$

To determine the position of the scattering, we compute, step by step, the optical thickness at the frequency of the photon $\tau$ crossed by the photon:

$$
\tau+d \tau=\tau+\sigma_{i, r e f} \sqrt{\frac{T_{r e f}}{T(r)}} \times e^{-x^{2} \frac{T_{i}}{T(\mathbf{r})}} n(r) \times p_{i}(r) \times d s(r),
$$

where $d s(r)$ is the step size (varying from $0.5 \mathrm{~km}$ at low altitudes to $10 \mathrm{~km}$ at high altitudes), the term in the exponential is the local normalized frequency, which depends on temperature through the Doppler width, $T_{i}$ is the temperature at the position where the photon has been emitted $\left(T_{i}=T_{\text {ref }}\right.$ before the first scattering), $T(r)$ and $n(r)$ are the local temperature and oxygen density, respectively, $\sigma_{i, r e f}$ is the cross section at the center of the line $i$ for the reference temperature $T_{\text {ref }}$, and the root square factor describes the variations of this cross section with the temperature.

[45] Value $p_{i}(r)$ is the percentage of oxygen in the ground state susceptible to absorbing the photons emitted in the line $i$. This percentage depends on the temperature as follows:

$$
p_{i}(\mathbf{r})=\frac{g_{i} e^{-\frac{\Delta E_{i}}{k T(\mathbf{r})}}}{\sum_{k=1}^{3} g_{k} e^{-\frac{\Delta E_{k}}{k T(\mathbf{r})}}}
$$

where

$$
\Delta E_{i}=\frac{h c}{\lambda_{0,1}}-\frac{h c}{\lambda_{0, i}}
$$

$h$ is the Planck's constant, $c$ is the light velocity, $\lambda_{0, i}$ is the wavelength of the $i$ th line of the triplet $(130.217,130.486$, and $130.603 \mathrm{~nm}), k$ is the Boltzmann's constant, and $g_{i}$ are the relative populations for an infinite temperature [Strickland and Donahue, 1970].

[46] We also estimate the optical thickness due to the absorption of $\mathrm{CO}_{2}$ by

$$
\tau_{a b s}+d \tau_{a b s}=\tau_{a b s}+\sigma_{a b s, i} \times n_{C O_{2}}(r) \times d s(r),
$$

where $n_{c o 2}(r)$ is the $\mathrm{CO}_{2}$ density and $\sigma_{a b s, i}$ is the absorption cross section at the wavelength $\lambda_{i}$. The weight $W$ of the photons initially equal to 1 is reduced by a factor $\exp \left(-\tau_{a b s}\right)$ at each step.

[47] We neglect the variation of the $\mathrm{CO}_{2}$ absorption cross section with temperatures. It only depends on the line. We follow the photon until $\tau=\tau_{s}$. If the photon crosses the limits of the described region before reaching this equality, we simulate a new solar photon; otherwise, we compute the scattering. When we have a scattering in $(r, \alpha)$, we assume that a photon is emitted at the same position. We assume an isotropic scattering, so the new direction of the photon is given by

$$
\begin{aligned}
& \varphi=2 \pi r_{\varphi} \\
& \theta=\arccos \left(1-2 r_{\theta}\right),
\end{aligned}
$$

where $r_{\phi}$ and $r_{\theta}$ are random numbers between 0 and 1 , and $\phi$ and $\theta$ are the two angles that define the new direction. The definition of these angles is given in Figure A1. Note that $\theta$ is the scattering angle. The line of the emitted photon is determined thanks to the relative population of the ground state (equation (A7)), and the accurate frequency in the line is estimated from the algorithm of Lee [1977] assuming a partial frequency redistribution. The basic method for the algorithm is the following.

[48] The only atoms that can absorb the photons are those that "see" the photons with the frequency $\nu_{0, i}$, that is, those whose velocity component $V_{1}$ along the direction of the incident photons follows

$$
\nu_{0, i}=\left(1-\frac{V_{1}}{c}\right) \times \nu
$$

Equation (A11) can be rewritten by replacing $\nu$ with the normalized frequency:

$$
x\left(\Delta \nu_{D, i}\left(T_{i}\right)-\frac{V_{1}}{c} \Delta \nu_{D, i}\left(T_{i}\right)\right)=\frac{\nu_{0, i} \times V_{1}}{c} .
$$

The second term on the left is negligible. We normalize the component of the atom velocity by

$$
V_{1}=V_{t h}(r) \times u_{1}
$$




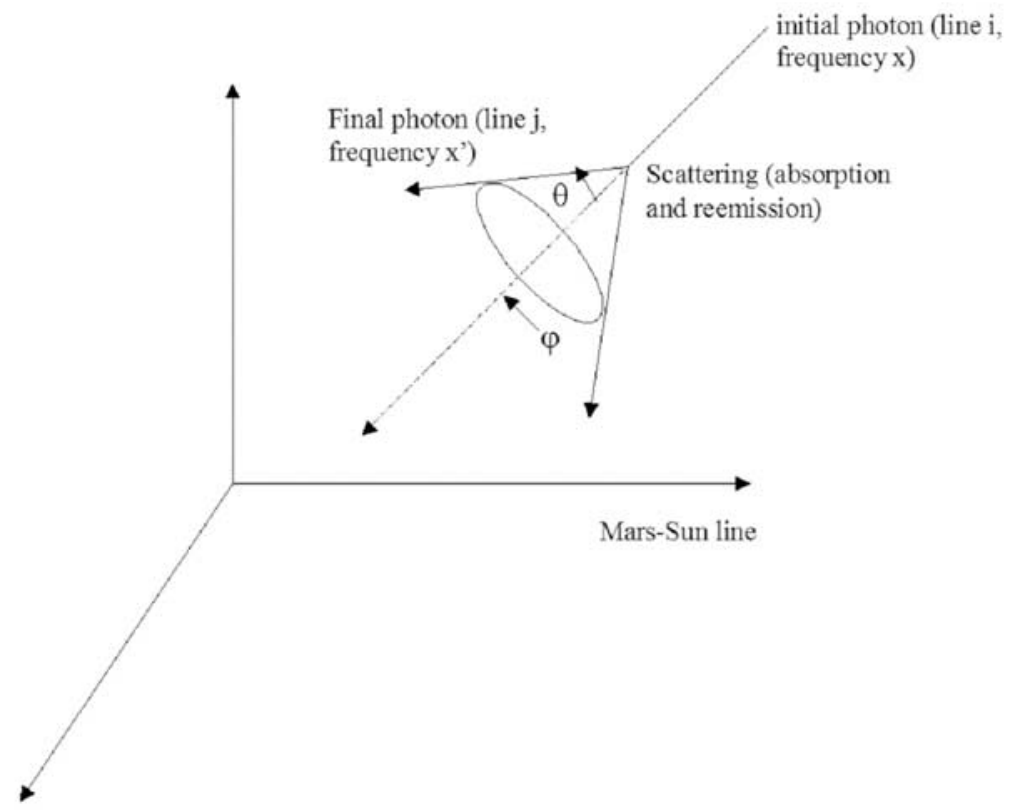

Figure A1. Geometry of the scattering (see Appendix A).

where $V_{t h}$ is the local thermal velocity. We can write

$$
\frac{V_{t h}}{\Delta \nu_{D}\left(T_{i}\right)}=\frac{c}{\nu_{0, i}} \frac{\sqrt{2 k T(r) / m}}{\sqrt{2 k T_{i} / m}}=\frac{c}{\nu_{0, i}} \sqrt{\frac{T(r)}{T_{i}}},
$$

where $T(r)$ is the local temperature and $T_{i}$ is the temperature at the position at which the photon incident has been emitted (exospheric temperature before the first scattering).

[49] Thus from equation (A12), we derive

$$
u_{1}=x \times \sqrt{\frac{T_{i}}{T(r)}} .
$$

We note $u_{2}$ is the atom-normalized velocity component orthogonal to the direction of propagation. This velocity is determined randomly by assuming a Gaussian velocity function distribution (even in the exosphere, which is probably not the case). We compute the velocity with the Box and Muller algorithm [Box and Muller, 1958]. If we consider that the new photon is emitted in the line $k$, the frequency of the photon in the referential frame of the atom will be $\nu_{0, k}$.

[50] If $u^{\prime}$ is the component of the atom-normalized velocity in the direction of propagation of the emitted photon, we have

$$
u^{\prime}=u_{1} \cos (\theta)+u_{2} \sin (\theta) .
$$

The frequency $\nu^{\prime}$ of the emitted photon in the referential frame (referential of the atmosphere) will follow the relation (A17):

$$
\nu_{0, k}=\left(1-\frac{u^{\prime} \times V_{t h}}{c}\right) \times \nu^{\prime}
$$

[51] Equation (A17) can be rewritten by replacing $\nu$ with the normalized frequency to provide

$$
x^{\prime}\left(\Delta \nu_{D, i}(T(r))-\frac{u^{\prime} \times V_{t h}}{c} \Delta \nu_{D, i}(T(r))\right)=\frac{\nu_{0, k} \times V_{t h}}{c} u^{\prime},
$$

which is the same equation as equation (A12), but this time the temperature defining the Doppler width is $T(r)$. Neglecting the second term on the left, equation (A18) is reduced to

$$
x^{\prime}=u^{\prime}=u_{1} \cos (\theta)+u_{2} \sin (\theta) .
$$

[52] Thereafter, we can estimate the contribution of the emitted photon to the simulated primary (for the first scattering) or multiple emission volume rate at the points of the spatial and frequency grids. The new photon is followed, and the same cycle is repeated. After each scattering, we update the volume emission of multiple scattering simulated. The process is stopped when the photon crosses the boundaries of the model, or when its weight becomes lower than $1 \times 10^{-9}$. The link between the real volume emission rate and the volume emission rate simulated is given by

$$
S_{i}^{\text {real }}(\mathbf{r}, x)=\frac{S_{\text {inc }} \times G}{V_{\text {cell }}(r) \cdot \Delta x \cdot N_{\text {photon }} \Delta \lambda_{D}(r)} \sum_{\text {photon }} W_{\text {photon }, i}^{\text {sim }}(\mathbf{r}, x),
$$

where $S_{i n c}$ is the surface area of the disk where solar photons are issued, $V_{\text {cell }}$ is the efficiency volume around the considered spatial point [Quémerais, 2000], $\Delta x$ is the frequency grid step, $N_{\text {photon }}$ is the total number of photons simulated, $\Sigma W_{\text {photon, } i}^{\text {sim }}(\mathbf{r}, x)$ is the emission volume rate simulated at the 
spatial grid point $\mathbf{r}$ and the frequency $x$ of the line $i$, and $G$ is the total solar flux in the three efficient lines.

[53] Once the emission volume rates have been calculated, we compute the intensity integrated on the line of sight for each observation for each model using the formal solution of the radiative transfer given by equation (A21). The only changes for one observation to another are the geometry of the line of sight (the direction $\Omega$ of the line of sight and the position of the spacecraft $\mathbf{r}$ ) and the value of the solar flux at $130.4 \mathrm{~nm}$ :

$$
\begin{aligned}
I(\mathbf{r}, \boldsymbol{\Omega})= & \frac{1}{4 \pi} \sum_{i=1}^{i=3} \int_{0}^{\infty} \int_{-\infty}^{\infty} S_{i}^{\text {real }}(\mathbf{r}+s \boldsymbol{\Omega}, \lambda) \\
& \cdot e^{-\tau_{\lambda, i}(\mathbf{r}+s \boldsymbol{\Omega}, \mathbf{r})} e^{-\tau_{C O_{2}, i}(\mathbf{r}+s \boldsymbol{\Omega}, \mathbf{r})} d \lambda d s .
\end{aligned}
$$

\section{Appendix B: Photoelectron Source}

[54] The variation of impact frequency of the photoelectrons $g_{p e}$ with the column density $N$ is based on the work of Stewart [1970] in the terrestrial case and is given by

$$
g_{p e}\left(N ; \mathrm{cm}^{-2}\right)=\frac{1}{B_{0}+B_{1}\left(N / 10^{17}\right)+B_{2}\left(N / 10^{17}\right)^{2}} .
$$

$B_{1}$ and $B_{2}$ have been chosen to reproduce the variations given in Figure 4 of Strickland et al. [1973]. $B_{0}$ is equal to $1 / g(0)$. We assume a linear relation between $g(0)$ and the solar index $F_{10.7}$ at Mars. The normalization factor $g(0)$ is the value estimated by Stewart et al. [1992]: $1.7 \times 10^{-7} \mathrm{~s}^{-1}$ for $F_{10.7}=54$.

[55] The scheme of the model for describing the multiple scattering due to photoelectron impacts is the same as that used to describe the solar source. Twenty million photons emitted by electronic impact are followed. The only difference comes from the computation of the photons before the first scattering. The position of the photons emitted (before the first scattering) is given by solving the following equation:

$$
\int_{0}^{\varphi} \int_{0}^{\mu} \int_{r_{\min }}^{r}\left(\frac{S_{0}^{p e}(\mu, r)}{W} r^{2} d r\right) d \mu d \varphi=\alpha \int_{0}^{2 \pi} \int_{0}^{1} \int_{r_{\min }}^{r_{\max }}\left(\frac{S_{0}^{p e}(\mu, r)}{W} r^{2} d r\right) d \mu d \varphi
$$

where $S_{0}^{p e}$ is the production volume rate, defined in equation (1), $W$ is the weight of the photons, which means the number of real photons represented by one test particle, $r$ is the distance from the center of Mars (assumed spherical), $\mu=\cos (\mathrm{SZA}), \varphi$ is the second spherical coordinate, and $\alpha$ is a random number between $[0,1]$. To simplify this calculation, we assume $W=$ $S_{0}^{p e} \times V_{\text {tot }}$, where $V_{\text {tot }}$ is the volume between $r_{\min }$ and $r_{\max }$. Under this assumption, the position of the first photon $(r, \mu, \phi)$ is given by

$$
\begin{aligned}
r & =\sqrt[3]{r_{\text {min }}^{3}+\alpha_{1}\left(r_{\text {max }}^{3}-r_{\text {min }}^{3}\right)} \\
\mu & =1-\alpha_{2} \\
\varphi & =\alpha_{3} \times 2 \pi
\end{aligned}
$$

The direction of the photon emitted is assumed to be isotropic; the line of the emitted photon is determined thanks to the relative population of the ground state at the local temperature (equation (A7)) and the frequency using the Box and Muller algorithm [Box and Muller, 1958]. The optical thickness before scattering is determined by using equation (A5).

[56] If the weight of the photon is higher than $10^{-9} \mathrm{~V}_{\text {tot }}$, the primary emission volume rate is updated, meaning that photons produced at a position where $S_{0}^{p e}<10^{-9} \mathrm{~cm}^{-3} \mathrm{~s}^{-1}$ are not taken into account. Each photon is followed until it crosses the upper limit or the lower limit or until its weight becomes lower than $1 \times 10^{-9} \times V_{t o t}$; the scheme used for multiple scattering is the same as that for the solar source.

[57] Then the spectral production volume rates are normalized by

$$
S_{p e, i}^{\text {real }}(\mathbf{r}, \lambda)=\left(\frac{P_{\text {real }}}{P_{\text {sim }}}\right) \frac{\sum_{\text {photon }} W_{\text {photons }, i}(\mathbf{r}, x)}{V_{\text {cell }}(r) \times \Delta x \times \Delta \lambda_{D}(r)},
$$

where $P_{\text {real }}$ is the real total production in $\mathrm{s}^{-1}$ for the atmospheric model (given by the right term of equation (B2) with $\alpha=1$ and $W=1$ ) and $P_{\text {sim }}$ is the total production simulated, which means the sum of the weight of all the photons emitted. The sum symbol corresponds to the sum of the weight of the photons emitted at the position $\mathbf{r}$ with a normalized frequency $x$ in the line $i$.

[58] Acknowledgments. Mars Express is a space mission from the European Space Agency (ESA). We wish to express our gratitude to all ESA members who participated in this successful mission. We would like to thank E. Dimarellis, A. Reberac, and J.-F. Daloze for planning the observations and for their help in the data processing. We thank our collaborators at the three institutes for the design and fabrication of the instrument (Service d'Aeronomie/France, BIRA/Belgium, and IKI/Moscow) and in particular Emiel Vanraansbeck at BIRA for careful mechanical design and fabrication. We wish to thank CNRS and CNES for financing SPICAM in France. In addition, we thank R. Gladstone for reviewing the grammatical aspects of this paper.

\section{References}

Banks, P. M., and G. Kockarts (1973), Aeronomy Part B, Elsevier, New York.

Barklem, P. S. (2007), Electron-impact excitation of neutral oxygen, Astron. Astrophys., 462, 781-788, doi:10.1051/0004-6361:20066341.

Barth, C. A., C. W. Hord, J. B. Pearce, K. K. Kelly, G. P. Anderson, and A. I. Stewart (1971), Mariner 6 and 7 ultraviolet spectrometer experiment: Upper atmosphere data, J. Geophys. Res., 76, 2213-2227, doi:10.1029/ JA076i010p02213.

Barthelemy, M., G. Gronoff, J. Lilensten, and J.-Y. Chaufray (2008), Radiative transfer of the oxygen $130 \mathrm{~nm}$ triplet through the atmosphere of Mars and Venus, Geophys. Res. Abstr., 10, EGU2008-A-09456, SRef-ID: 1607-7962/gra/EGU2008-A-09456.

Bertaux, J.-L., J. E. Blamont, V. M. Lupine, V. G. Kurt, N. N. Romanova, and A. S. Smirnov (1981), Venera 11 and Venera 12 observations of EUV emissions from the upper atmosphere of Venus, Planet. Space Sci., 29, 149-166, doi:10.1016/0032-0633(81)90029-5.

Bertaux, J.-L., et al. (2006), SPICAM on Mars Express: Observing modes and overview of the spectrometer data and scientific results, J. Geophys. Res., 111, E10S90, doi:10.1029/2006JE002690.

Bougher, S. W., R. G. Roble, E. C. Ridley, and R. E. Dickinson (1990), The Mars thermosphere: 2. General circulation with coupled dynamics and composition, J. Geophys. Res., 95, 14,811-14,827, doi:10.1029/ JB095iB09p14811.

Bougher, S. W., D. M. Hunten, and R. G. Roble (1994), $\mathrm{CO}_{2}$ cooling in terrestrial planet thermospheres, J. Geophys. Res., 99, 14,609-14,622, doi:10.1029/94JE01088.

Bougher, S. W., J. M. Murphy, and R. M. Haberle (1997), Dust storm impacts on the Mars upper atmosphere, Adv. Space Res., 19, 1255-1260, doi:10.1016/S0273-1177(97)00278-0. 
Bougher, S. W., S. Engel, R. G. Roble, and B. Foster (1999), Comparative terrestrial planet thermospheres: 2. Solar cycle variation of global structure and winds at equinox, J. Geophys. Res., 104, 16,591-16,611, doi:10.1029/1998JE001019.

Bougher, S. W., S. Engel, R. G. Roble, and B. Foster (2000), Comparative terrestrial planet thermospheres: 3. Solar cycle variation of global structure and winds at solstices, J. Geophys. Res., 105, 17,669-17,692, doi:10.1029/1999JE001232.

Box, G., and M. E. Muller (1958), A note on the generation of random normal deviate, Ann. Math. Stat., 29, 610-611, doi:10.1214/aoms/ 1177706645

Broadfoot, A. L., S. Kumar, M. J. S. Belton, and M. B. McElroy (1974), Ultraviolet observations of Venus from Mariner 10: Preliminary results, Science, 183, 1315-1318, doi:10.1126/science.183.4131.1315.

Chamberlain, J. W. (1963), Planetary coronae and atmospheric evaporation, Planet. Space Sci., 11, 901-960, doi:10.1016/0032-0633(63)90122-3.

Chaufray, J.-Y., R. Modolo, F. Leblanc, G. Chanteur, R. E. Johnson, and J. G. Luhmann (2007), Mars solar wind interaction: Formation of the Martian corona and atmospheric loss to space, J. Geophys. Res., 112, E09009, doi:10.1029/2007JE002915.

Chaufray, J.-Y., J.-L. Bertaux, F. Leblanc, and E. Quémerais (2008), Observation of the hydrogen corona by SPICAM on Mars Express, Icarus, 195, 598-613, doi:10.1016/j.icarus.2008.01.009.

Chubb, T. A., E. T. Byram, I. Friedman, and J. E. Kupperian (1958), The use of radiation absorption and luminescence in upper air density measurements, Ann. Geophys., 14, 109-116.

Cipriani, F., F. Leblanc, and J.-J. Berthelier (2007), Martian corona: Nonthermal sources of hot heavy species, J. Geophys. Res., 112, E07001, doi:10.1029/2006JE002818.

Cotton, D. M., G. R. Gladstone, and S. Chakrabarti (1993), Sounding rocket observation of a hot atomic oxygen geocorona, J. Geophys. Res., 98, 21,651-21,657, doi:10.1029/93JA02268.

Feldman, P. D., E. B. Burgh, S. T. Durrance, and A. F. Davidsen (2000), Far-ultraviolet spectroscopy of Venus and Mars at 4A resolution with the Hopkins Ultraviolet Telescope on Astro-2, Astrophys. J., 538, 395-400, doi:10.1086/309125.

Fox, J. L. (2003), The effect of $\mathrm{H}_{2}$ on the Martian ionosphere: Implications for atmospheric evolution, J. Geophys. Res., 108(A6), 1223, doi:10.1029/ 2001JA000203

Fox, J. L., and A. Dalgarno (1979), Ionization, luminosity and heating of the upper atmosphere of Mars, J. Geophys. Res., 84, 7315-7333, doi:10.1029/JA084iA12p07315.

Gladstone, G. R. (1992), Solar OI 1304-A triplet line profiles, J. Geophys Res., 97, 19,519-19,525, doi:10.1029/92JA00991.

Hanson, W. B., S. Sanatani, and D. R. Zuccaro (1977), The Martian ionosphere as observed by the Viking retarding potential analyzers, J. Geophys. Res., 82, 4351-4363, doi:10.1029/JS082i028p04351.

Hubert, B., J.-C. Gerard, D. M. Cotton, D. V. Bisikalo, and V. I. Shematovich (1999), Effect of hot oxygen on thermospheric O I UV airglow, J. Geophys. Res., 104, 17,139-17,143, doi:10.1029/1999JA900140.

Hunten, D. M. (1973), The escape of light gases from planetary atmospheres, J. Atmos. Sci., 30, 1481-1494, doi:10.1175/1520-0469[1973]030 $<1481$ :TEOLGF $>2.0 . \mathrm{CO} ; 2$

Keating, G. M., et al. (1998), The structure of the upper atmosphere of Mars: In situ accelerometer measurements from Mars Global Surveyor, Science, 279, 1672-1676, doi:10.1126/science.279.5357.1672.

Krasnopolsky, V. A. (2002), Mars' upper atmosphere and ionosphere at low, medium and high solar activities: Implications for evolution of water, J. Geophys. Res., 107(E12), 5128, doi:10.1029/2001JE001809.
Krestyanikova, M. A., and V. I. Shematovich (2005), Stochastic models of hot planetary and satellite coronas: A photochemical source of hot oxygen in the upper atmosphere of Mars, Sol. Syst. Res., 39, 22-32.

Leblanc, F., J. Y. Chaufray, J. Lilensten, O. Witasse, and J. L. Bertaux (2006), Martian dayglow as seen by the SPICAM UV spectrograph on Mars Express, J. Geophys. Res., 111, E09S11, doi:10.1029/2005JE002664.

Leblanc, F., J.-Y. Chaufray, J. Lilenstein, O. Witasse, J.-L. Bertaux, and the SPICAM Team (2007), On Martian nitrogen dayglow emission observed by SPICAM UV spectrograph/Mars Express, Geophys. Res. Lett., 34, L02206, doi:10.1029/2006GL028437.

Lee, J.-S. (1977), Simulation of emission frequencies from angle-dependent partial frequency redistributions, Astrophys. J., 218, 857-865, doi:10.1086/ 155741

Mazarico, E., M. T. Zuber, F. G. Lemoine, and D. E. Smith (2007), Martian exospheric density using Mars Odyssey radio tracking data, J. Geophys. Res., 112, E05014, doi:10.1029/2006JE002734.

Meier, R. R., and J.-S. Lee (1982), An analysis of the OI 1304 A dayglow using a Monte Carlo resonant scattering model with partial frequency redistribution, Planet. Space Sci., 30, 439, doi:10.1016/0032-0633(82) 90053-8.

Moos, H. W., and G. J. Rottman (1971), O I and H I emissions from the upper atmosphere of Venus, Astrophys. J., 169, L127, doi:10.1086/180827.

Nagy, A. F., and T. E. Cravens (1988), Hot oxygen atoms in the upper atmospheres of Venus and Mars, Geophys. Res. Lett., 15, 433-435, doi:10.1029/GL015i005p00433

Quémerais, E. (2000), Angle dependent partial frequency redistribution in the interplanetary medium at Lyman $\alpha$, Astron. Astrophys., 358,353-367.

Rottman, G. J., N. W. Thomas, and W. McClintock (2006), SORCE solar UV irradiance results, Adv. Space Res., 37, 201-208.

Shematovich, V. I., D. V. Bisikalo, J.-C. Gerard, C. Cox, S. W. Bougher, and F. Leblanc (2008), Monte Carlo model of electron transport for the calculation of Mars dayglow emissions, J. Geophys. Res., 113, E02011, doi:10.1029/2007JE002938.

Stewart, A. I. (1970), Photoionization coefficients and photoelectron impact excitation efficiencies in the daytime ionosphere, J. Geophys. Res., 75 6333-6338, doi:10.1029/JA075i031p06333.

Stewart, A. I. F. (1987), A revised time dependent model of the Martian atmosphere for use in orbit lifetime and sustenance studies: Final report, JPL PO NQ-802429, Jet Propul. Lab., Pasadena, Calif.

Stewart, A. I., M. J. Alexander, R. R. Meier, L. J. Paxton, S. W. Bougher, and C. G. Fesen (1992), Atomic oxygen in the Martian thermosphere, J. Geophys. Res., 97, 91-102, doi:10.1029/91JA02489.

Strickland, D. J., and T. M. Donahue (1970), Excitation and radiative transport of OI $1304 \AA$ resonance radiation, I: The dayglow, Planet. Space Sci., 18, 661-689, doi:10.1016/0032-0633(70)90049-8.

Strickland, D. J., G. E. Thomas, and P. R. Sparks (1972), Mariner 6 and 7 ultraviolet spectrometer experiment: Analysis of the OI 1304- and 1356-A emissions, J. Geophys. Res., 77, 4052-4068, doi:10.1029/JA077i022p04052.

Strickland, D. J., A. I. Stewart, C. A. Barth, C. W. Hord, and A. L. Lane (1973), Mariner 9 Ultraviolet Spectrometer Experiment: Mars atomic oxygen 1304-A emission, J. Geophys. Res., 78, 4547-4559, doi:10.1029/JA078i022p04547.

Zipf, E. C., and P. W. Erdman (1985), Electron impact excitation of atomic oxygen: Revised cross sections, J. Geophys. Res., 90, 11,087-11,090, doi:10.1029/JA090iA11p11087.

J. L. Bertaux, F. Leblanc, and E. Quémerais, Laboratoire Atmospheres, Milieu, Observations Spatiales, IPSL, CNRS, Reduit de Verrieres, BP 3, Route des Gatines, F-91371 Verrières-le-Buisson CEDEX, France.

J. Y. Chaufray, Southwest Research Institute, P.O. Drawer 28510, San Antonio, TX 78228-0510, USA. (jchaufray@swri.org) 